\title{
Galactic rotation curves versus ultralight dark matter: Implications of the soliton-host halo relation
}

\author{
Nitsan Bar, ${ }^{1}$ Diego Blas, ${ }^{2,3}$ Kfir Blum, ${ }^{1,2}$ and Sergey Sibiryakov ${ }^{2,4,5}$ \\ ${ }^{1}$ Weizmann Institute of Science, 234 Herzl Street, Rehovot 7610001, Israel \\ ${ }^{2}$ Theory department, CERN, CH-1211 Geneve 23, Switzerland \\ ${ }^{3}$ Theoretical Particle Physics and Cosmology Group, Department of Physics, \\ King's College London, Strand, London WC2R 2LS, United Kingdom \\ ${ }^{4}$ LPPC, IPHYS, Ecole Politechnique Fédérale de Lausanne, CH-1015 Lausanne, Switzerland \\ ${ }^{5}$ Institute for Nuclear Research of the Russian Academy of Sciences, 60th October Anniversary Prospect, \\ 7a, 117312 Moscow, Russia
}

(Received 15 May 2018; published 31 October 2018)

\begin{abstract}
Bosonic ultralight dark matter (ULDM) would form cored density distributions at the center of galaxies. These cores, seen in numerical simulations, admit analytic description as the lowest energy bound state solution ("soliton") of the Schroedinger-Poisson equations. Numerical simulations of ULDM galactic halos find empirical scaling relations between the mass of the large-scale host halo and the mass of the central soliton. We discuss how the simulation results of different groups can be understood in terms of the basic properties of the soliton. Importantly, simulations imply that the energy per unit mass in the soliton and in the virialized host halo should be approximately equal. This relation lends itself to observational tests because it predicts that the peak circular velocity, measured for the host halo in the outskirts of the galaxy, should approximately repeat itself in the central region. Contrasting this prediction with the measured rotation curves of well-resolved nearby galaxies, we show that ULDM in the mass range $m \sim\left(10^{-22} \div 10^{-21}\right) \mathrm{eV}$, which has been invoked as a possible solution to the small-scale puzzles of $\Lambda \mathrm{CDM}$, is in tension with the data. We suggest that a dedicated analysis of the Milky Way inner gravitational potential could probe ULDM up to $m \lesssim 10^{-19} \mathrm{eV}$.
\end{abstract}

DOI: 10.1103/PhysRevD.98.083027

\section{INTRODUCTION}

Two forms of matter could provide the cosmological equation of state, required for dark matter (DM): nonrelativistic massive particles (e.g., WIMPs), or the classical background of an ultralight bosonic field oscillating around a minimum of its potential (e.g., axions or axionlike particles). On cosmological scales, WIMPs and ultralight dark matter (ULDM) behave similarly. However, on scales comparable to its de Broglie wavelength, ULDM behaves markedly different from WIMPs [1-13].

Axionlike particles with exponentially suppressed masses arise in the context of string theory [12,14-16]. They are produced by misalignment and for mass in the range $m \sim\left(10^{-22} \div 10^{-18}\right) \mathrm{eV}$ their cosmic abundance could naturally match the observed DM density.

ULDM with mass $m \sim\left(10^{-22} \div 10^{-21}\right) \mathrm{eV}$ is particularly motivated due to several puzzles, facing the standard WIMP paradigm on galactic scales (see, e.g., $[12,17]$ for recent

Published by the American Physical Society under the terms of the Creative Commons Attribution 4.0 International license. Further distribution of this work must maintain attribution to the author(s) and the published article's title, journal citation, and DOI. reviews). This range of $m$ is in some tension with the matter power spectrum, inferred from Ly- $\alpha$ forest analyses [18-22], which yields a bound $m \gtrsim 10^{-21} \mathrm{eV}$. Nevertheless, since the strongest Ly- $\alpha$ bound constraints come from the smallest and most nonlinear scales, where systematic effects are challenging, we believe it prudent to seek additional methods to constrain the model for $m \gtrsim 10^{-22} \mathrm{eV}$.

Numerical simulations $[6,7,10,11,13]$ show that ULDM forms cored density profiles in the inner region of galactic halos, roughly within the de Broglie wavelength,

$$
x_{d B} \approx 190\left(\frac{v}{100 \mathrm{~km} / \mathrm{s}}\right)^{-1}\left(\frac{m}{10^{-22} \mathrm{eV}}\right)^{-1} \mathrm{pc} .
$$

The core, referred to as "soliton" in the literature $[4,5,8,9]$, corresponds to a coherent quasi-stationary solution of the ULDM equations of motion and its density profile can be derived analytically. ${ }^{1}$ This match between analytic and numerical simulation results offers a unique opportunity to

\footnotetext{
${ }^{1}$ Strictly speaking, the soliton solution is only known numerically. With some abuse of the word, we refer to it here as analytical to emphasize that the solution can be found by integrating a simple differential equation in a procedure that takes $<1$ sec on a standard laptop.
} 
understand and extend the simulations, making the ULDM model potentially more predictive than the WIMP paradigm. For nearby dwarf galaxies $(v \sim 10 \mathrm{~km} / \mathrm{s})$ or Milky Way (MW)-like galaxies ( $v \sim 100 \mathrm{~km} / \mathrm{s}$ ), the soliton core could be resolved with current observational tools, offering a test of the model.

Attempts to detect, constrain, or fit ULDM soliton cores using galactic rotation velocity and velocity dispersion data were discussed in the literature [2,3,6-8,23-26]. Some of these analyses fitted a soliton profile to describe entire galaxies [2,3,23]. This exercise leads to small ULDM particle mass, $m \lesssim 10^{-23} \mathrm{eV}$, in strong tension with cosmological bounds [18-22]. Furthermore, numerical simulations $[6,7,10,11,13]$ have shown that galactic halos above a certain mass would exhibit a more complex structure, with the central soliton transiting into a large-scale host halo composed of an incoherent superposition of multiple scalar field wavepackets. Consequently, other works [8,24-26] analyzed galactic profiles using a soliton + host halo description.

A key question in comparing the soliton + host halo model to kinematical data is how to model the transition between the central soliton and the host halo. Previous phenomenological analyses [8,24-26] defined separate free parameters for the host halo and for the soliton, for each individual galaxy in the sample. On the numerical simulation side, several studies focused on finding a solitonhost halo relation $[6,10,11,13]$. The point in finding a soliton-host halo relation is, of course, that it could tie together the behavior of ULDM in the large-scale host halo to predict the central core with fewer free parameters.

In this work, we consider the soliton-host halo relations, reported by different numerical simulation groups $[6,7,10,11,13]$. Our first observation is that properties of the analytic soliton solution can provide important insight on the numerical results. We show that: (i) the soliton-host halo relation, reported in [13], essentially attributes the total energy (kinetic + gravitational) of the halo to the dominant soliton. This energetic dominance of the soliton is unlikely to hold for realistic galaxies above a certain size; (ii) the soliton-host halo relation, reported in [7], essentially equates the energy per unit mass in the soliton to the energy per unit mass in the virialized halo. This relation can apply to real galaxies.

Assuming the soliton-host halo relation of [7], we show that it leads to a prediction: the peak circular velocity characterizing the host halo on large scales (few kpc for typical $\left(10^{9} \div 10^{10}\right) \mathrm{M}_{\odot}$ galaxies) should repeat itself in the core on small scales $(\lesssim 1 \mathrm{kpc})$, insensitive to the details of the host halo density profile. This implies an observational constraint that can be tested without free parameters. Applying this test to high-resolution rotation curves of late-type galaxies from $[27,28]$, we find that ULDM in the mass range $m \sim\left(10^{-22} \div 10^{-21}\right) \mathrm{eV}$ is disfavored by the data. Baryonic physics is unlikely to cure the tension: the discrepancy between the predictions of ULDM (with the soliton-host halo relation) and the data are too large. In many galaxies in the sample we analyze, baryonic feedback would need to overcome a dark matter-to-baryon mass ratio of $\gtrsim 10: 1$, to destroy the soliton. As a result, if the solitonhost halo relation of $[6,7]$ holds for real systems, ULDM is disfavored below $m \sim 10^{-21} \mathrm{eV}$ and is unlikely to play a role in solving the small scale puzzles of $\Lambda \mathrm{CDM}$.

We also discuss observational imprints of the soliton in big galaxies, such as the MW. In this case, the shape of the soliton is modified due to the gravitational potential of baryonic matter and supermassive black hole (SMBH). Preliminary numerical simulations of ULDM halos with stars [29] indicate that in the presence of baryons, the soliton mass stays the same or increases compared to the soliton-host halo prediction of pure ULDM. If these results are confirmed, solitons formed by ULDM with masses $m \lesssim 10^{-19} \mathrm{eV}$ will produce order-one contribution into the mass budget of the inner MW. A dedicated analysis of inner MW kinematics-including simultaneous modeling of the baryonic mass and the soliton-could potentially test ULDM up to $m \lesssim 10^{-19} \mathrm{eV}$.

The outline of this paper is as follows. In Sec. II, we review some basic properties of the soliton. In Sec. III, we discuss the soliton-host halo relations found in simulations, and show that these relations can be understood in terms of fundamental properties of the soliton and the halo. The simulations of $[6,7]$ are considered in Sec. III A; they can be summarized by the statement $\left.(E / M)\right|_{\text {soliton }}=\left.(E / M)\right|_{\text {halo }}$, where $E$ is the total energy (kinetic + gravitational, within the virial radius, for the halo) and $M$ is the mass (again within the virial radius, for the halo). The simulations of [13] are considered in Sec. III B; their soliton-halo result can be summarized by the statement $\left.E\right|_{\text {soliton }}=\left.E\right|_{\text {halo }}$. We argue that this result is unlikely to represent realistic galaxies above a certain size.

In Sec. IV, adopting the soliton-host halo relation of [6,7], we work out its observational consequences. We show that in ULDM galaxies satisfying this relation, the rotation velocity in the inner core should be approximately as high as the peak rotation velocity in the outer part of the galaxy. In Sec. IV A, we compare this analysis to numerical profiles taken directly from the published simulation results, finding good agreement. In Sec. IV B, we compare this prediction to real galaxies from [27,28], finding tension for $m \sim$ $\left(10^{-22} \div 10^{-21}\right) \mathrm{eV}$. We show that the intrinsic scatter in the soliton-host halo relation does not resolve the discrepancy.

In Sec. V, we calculate how baryonic effects could modify the soliton solution. In Sec. VA, we consider a smooth fixed distribution of baryonic mass, deferring the case of a super-massive black hole to App. B. These results are applied to the MW in Sec. V B.

In Sec. VI, we compare our results to previous literature and discuss some caveats to our analysis. Sec. VII contains a summary of our results and open questions. 


\section{SOLITON PROPERTIES: ANALYTIC CONSIDERATIONS}

In this section, we review the relevant properties of the soliton that help to understand results from numerical simulations.

We consider a real, massive, free ${ }^{2}$ scalar field $\phi$, satisfying the Klein-Gordon equation of motion and minimally coupled to gravity. In the nonrelativistic regime, it is convenient to decompose $\phi$ as

$$
\phi(x, t)=\frac{1}{\sqrt{2} m} e^{-i m t} \psi(x, t)+\text { c.c. },
$$

with complex field $\psi$ that varies slowly in space and time, such that $|\nabla \psi| \ll m|\psi|$ and $|\dot{\psi}| \ll m|\psi|$. The field $\psi$ satisfies the Schroedinger-Poisson (SP) equations [32]

$$
\begin{gathered}
i \partial_{t} \psi=-\frac{1}{2 m} \nabla^{2} \psi+m \Phi \psi, \\
\nabla^{2} \Phi=4 \pi G|\psi|^{2} .
\end{gathered}
$$

We look for a quasi-stationary phase-coherent solution, described by the ansatz ${ }^{3}$

$$
\psi(x, t)=\left(\frac{m M_{\mathrm{pl}}}{\sqrt{4 \pi}}\right) e^{-i \gamma m t} \chi(x) .
$$

The ULDM mass density is

$$
\begin{aligned}
\rho & =\frac{\left(m M_{\mathrm{pl}}\right)^{2}}{4 \pi} \chi^{2} \\
& \approx 4.1 \times 10^{14}\left(\frac{m}{10^{-22} \mathrm{eV}}\right)^{2} \chi^{2} \mathrm{M}_{\odot} / \mathrm{pc}^{3} .
\end{aligned}
$$

The parameter $\gamma$ is proportional to the ULDM energy per unit mass. Validity of the nonrelativistic regime requires $|\gamma| \ll 1$. Since we are looking for gravitationally bound configurations, $\gamma<0$.

Assuming spherical symmetry and defining $r=m x$, the SP equations for $\chi$ and $\Phi$ are given by

$$
\begin{gathered}
\partial_{r}^{2}(r \chi)=2 r(\Phi-\gamma) \chi, \\
\partial_{r}^{2}(r \Phi)=r \chi^{2} .
\end{gathered}
$$

Finding the ground state solution amounts to solving Eqs. (7)-(8) subject to $\chi(r \rightarrow 0)=$ const, $\chi(r \rightarrow \infty)=0$, with no nodes. Given the boundary value of $\chi$ at $r \rightarrow 0$, the solution is found for a unique value of $\gamma$.

\footnotetext{
${ }^{2}$ Analyses of interacting fields can be found in, e.g., $[4,5,30,31]$.

${ }^{3} M_{\mathrm{pl}}=1 / \sqrt{G}$.
}

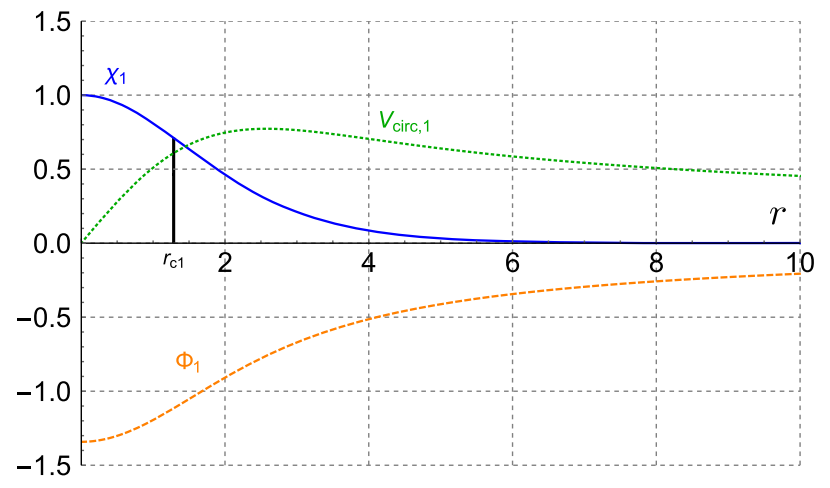

FIG. 1. Profile of the "standard" $\chi_{1}$ soliton with $\lambda=1$ (blue solid). We also show the corresponding gravitational potential (orange dashed) and circular velocity of a test particle (dotted green).

It is convenient to first solve Eqs. (7)-(8) with the boundary condition $\chi(0)=1$. Let us call this auxiliary solution $\chi_{1}(r)$, with $\gamma_{1}$. A numerical calculation gives $[4,5,8]$

$$
\gamma_{1} \approx-0.69,
$$

and the solution is plotted in Fig. 1. The mass of the $\chi_{1}$ soliton is

$$
\begin{aligned}
M_{1} & =\frac{M_{\mathrm{pl}}^{2}}{m} \int_{0}^{\infty} d r r^{2} \chi_{1}^{2}(r) \\
& \approx 2.79 \times 10^{12}\left(\frac{m}{10^{-22} \mathrm{eV}}\right)^{-1} \mathrm{M}_{\odot} .
\end{aligned}
$$

Its core radius, defined as the radius where the mass density drops by a factor of 2 from its value at the origin, is

$$
x_{c 1} \approx 0.082\left(\frac{m}{10^{-22} \mathrm{eV}}\right)^{-1} \text { pc. }
$$

Other solutions of Eqs. (7)-(8) can be obtained from $\chi_{1}(r), \Phi_{1}(r)$ by a scale transformation. That is, the functions $\chi_{\lambda}(r), \Phi_{\lambda}(r)$, together with the eigenvalue $\gamma_{\lambda}$, given by

$$
\begin{aligned}
\chi_{\lambda}(r) & =\lambda^{2} \chi_{1}(\lambda r), \\
\Phi_{\lambda}(r) & =\lambda^{2} \Phi_{1}(\lambda r), \\
\gamma_{\lambda} & =\lambda^{2} \gamma_{1},
\end{aligned}
$$

also satisfy Eqs. (7)-(8) with correct boundary conditions for any $\lambda>0$. The soliton mass and core radius for $\chi_{\lambda}$ are

$$
\begin{gathered}
M_{\lambda}=\lambda M_{1}, \\
x_{c \lambda}=\lambda^{-1} x_{c 1} .
\end{gathered}
$$

A mnemonic for the numerical value of $\lambda$ is given by 


$$
\lambda=3.6 \times 10^{-4}\left(\frac{m}{10^{-22} \mathrm{eV}}\right)\left(\frac{M_{\lambda}}{10^{9} \mathrm{M}_{\odot}}\right) .
$$

The product of the soliton mass and core radius is independent of $\lambda$,

$$
M_{\lambda} x_{c \lambda} \approx 2.27 \times 10^{8}\left(\frac{m}{10^{-22} \mathrm{eV}}\right)^{-2} \mathrm{kpc} \mathrm{M}_{\odot} .
$$

Formally, solutions exist for any positive value of $\lambda$ and hence for any soliton mass. However, if we select $\lambda \gtrsim 1$ we reach $\left|\gamma_{\lambda}\right|>1$, outside of the regime of validity of the nonrelativistic approximation. Thus, self-consistent solutions are limited to $\lambda \ll 1$ and their eigenvalue $\left|\gamma_{\lambda}\right|=\lambda^{2}\left|\gamma_{1}\right| \ll 1$, consistent with the nonrelativistic approximation.

The energy in an arbitrary nonrelativistic ULDM configuration is

$$
E=\int d^{3} x\left(\frac{|\nabla \psi|^{2}}{2 m^{2}}+\frac{\Phi|\psi|^{2}}{2}\right)=E_{k}+E_{p},
$$

with kinetic (potential) energy $E_{k}\left(E_{p}\right)$. For the ansatz (5), integrating by parts and using Eqs. (3)-(5), we have

$$
E=\frac{1}{3} M \gamma
$$

Note that spherical symmetry is not needed for Eq. (20) to hold.

Considering the $\chi_{\lambda}$ solitons, we find $E_{p, \lambda}=-2 E_{k, \lambda}=$ $2 E_{\lambda}$ with

$$
\begin{gathered}
E_{\lambda} \approx-0.476 \lambda^{3} \frac{M_{\mathrm{pl}}^{2}}{m}, \\
M_{\lambda} \approx 2.06 \lambda \frac{M_{\mathrm{pl}}^{2}}{m} .
\end{gathered}
$$

This leads to a relation for an isolated soliton [4,5],

$$
\frac{M_{\lambda}}{\left(M_{\mathrm{pl}}^{2} / m\right)} \approx 2.64\left|\frac{E_{\lambda}}{\left(M_{\mathrm{pl}}^{2} / m\right)}\right|^{\frac{1}{3}} .
$$

Another useful relation gives the energy per unit mass from the scaling parameter $\lambda$,

$$
\frac{\left|E_{\lambda}\right|}{M_{\lambda}} \approx 0.23 \lambda^{2}
$$

which can also be written as

$$
M_{\lambda} \approx 4.3\left(\frac{\left|E_{\lambda}\right|}{M_{\lambda}}\right)^{\frac{1}{2}} \frac{M_{\mathrm{pl}}^{2}}{m} .
$$

The circular velocity curve for a test particle in the soliton gravitational potential is given by

$$
V_{\text {circ }, \lambda}^{2}(r)=r \partial_{r} \Phi_{\lambda}(r) \text {. }
$$

The circular velocity rises as $V_{\text {circ }, \lambda} \propto r$ at small $r$ and decreases as $V_{\text {circ, } \lambda} \propto r^{-\frac{1}{2}}$ at large $r$, see Fig. 1. The peak of $V_{\text {circ }}$ is obtained at

$$
\begin{aligned}
x_{\text {peak }, \lambda} & \approx 0.16 \lambda^{-1}\left(\frac{m}{10^{-22} \mathrm{eV}}\right)^{-1} \mathrm{pc} \\
& \approx 460\left(\frac{m}{10^{-22} \mathrm{eV}}\right)^{-2}\left(\frac{M_{\lambda}}{10^{9} \mathrm{M}_{\odot}}\right)^{-1} \mathrm{pc},
\end{aligned}
$$

and the peak velocity is

$$
\begin{aligned}
\max V_{\text {circ }, \lambda} & \approx 2.3 \times 10^{5} \lambda \mathrm{km} / \mathrm{s} \\
& \approx 83\left(\frac{m}{10^{-22} \mathrm{eV}}\right)\left(\frac{M_{\lambda}}{10^{9} \mathrm{M}_{\odot}}\right) \mathrm{km} / \mathrm{s} .
\end{aligned}
$$

\section{MAKING CONTACT WITH NUMERICAL SIMULATIONS}

We now discuss results from the numerical simulations of three different groups, Refs. [6,7], Ref. [13], and Refs. [10,11].

The first point to note is that soliton configurations, in a form close to the idealized form discussed in Sec. II, actually occur dynamically in the central region of the halo in the numerical simulations. ${ }^{4}$ In Fig. 2, we collect representative density profiles from Ref. [6] (blue), Ref. [13] (orange), and Ref. [10] (green). We refer to those papers for more details on the specific set-ups in each simulation. To make Fig. 2 , in each case, we find the $\lambda$ parameter that takes the numerical result into the $\chi_{1}$ soliton, rescale the numerical result accordingly and present it in comparison with the analytic $\chi_{1}^{2}(r)$ profile.

While different groups agree that solitons form in the centers of halos, they do not appear to agree on the matching between the inner soliton profile and the host halo. Refs. [6,7] and Ref. [13] reported scaling relations between the central soliton and the host halo. As we show below, the scaling relations found by both groups are connected to properties of a single, isolated, self-gravitating soliton (part of these observations were made in $[10,11]$ ).

\section{A. Soliton vs host halo: The simulations of Ref. [6,7]}

At cosmological redshift $z=0$, the numerical simulations of $[6,7]$ yield approximately NFW-like halos which transit, in the central region, into a core with core radius and mass density

$$
x_{c} \approx 160\left(\frac{M_{h}}{10^{12} \mathrm{M}_{\odot}}\right)^{-\frac{1}{3}}\left(\frac{m}{10^{-22} \mathrm{eV}}\right)^{-1} \mathrm{pc},
$$

\footnotetext{
${ }^{4}$ The first simulations of cosmological ULDM galaxies [33] did not have sufficient resolution to resolve the central core.
} 


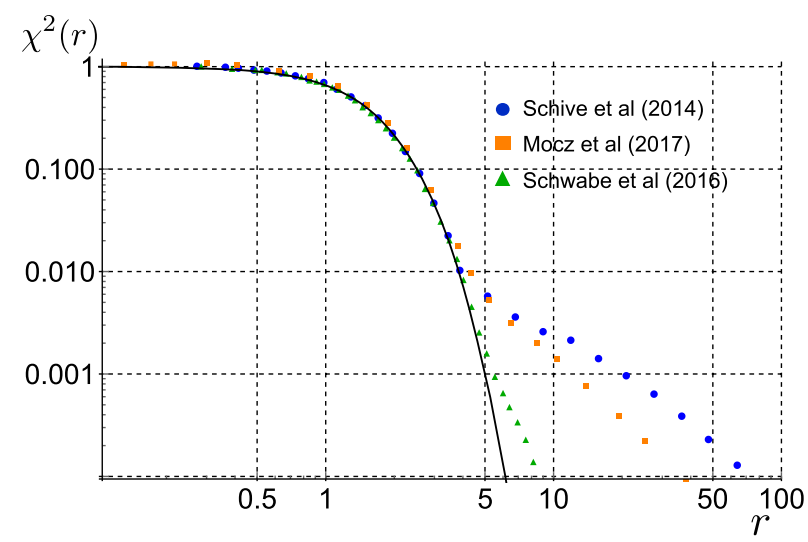

FIG. 2. Review of results from numerical simulations by different groups. Markers show density profiles of simulated halos from Schive et al. [6] (blue circles), Mocz et al. [13] (orange squares), and Schwabe et al. [10] (green triangles). The central regions of the halos are described by the soliton (solid line).

$$
\rho(x) \approx \frac{190\left(\frac{m}{10^{-22} \mathrm{eV}}\right)^{-2}\left(\frac{x_{c}}{100 \mathrm{pc}}\right)^{-4}}{\left(1+0.091\left(\frac{x}{x_{c}}\right)^{2}\right)^{8}} \mathrm{M}_{\odot} \mathrm{pc}^{-3},
$$

where $M_{h}$ is the virial mass of the host halo. As noted in [6-8], Eqs. (29)-(30) are an excellent numerical fit for a soliton $\chi_{\lambda}$. The mass of this soliton is

$$
M \approx 1.4 \times 10^{9}\left(\frac{m}{10^{-22} \mathrm{eV}}\right)^{-1}\left(\frac{M_{h}}{10^{12} \mathrm{M}_{\odot}}\right)^{\frac{1}{3}} \mathrm{M}_{\odot}
$$

so its $\lambda$ parameter is

$$
\lambda \approx 4.9 \times 10^{-4}\left(\frac{M_{h}}{10^{12} \mathrm{M}_{\odot}}\right)^{\frac{1}{3}} .
$$

Note that Eq. (31) is applicable only as long as the halo exceeds a minimal mass,

$$
M_{h, \min } \sim 5.2 \times 10^{7}\left(\frac{m}{10^{-22} \mathrm{eV}}\right)^{-3 / 2} \mathrm{M}_{\odot} .
$$

Smaller mass halos would be dominated by the soliton.

Reference [7] showed that Eq. (31) is consistent with the relation,

$$
M_{c} \approx \alpha\left(\frac{\left|E_{h}\right|}{M_{h}}\right)^{\frac{1}{2}} \frac{M_{\mathrm{pl}}^{2}}{m},
$$

where $M_{c}$ is the core mass (mass within $x<x_{c}$ ); $M_{h}, E_{h}$ are the virial mass and energy of the simulated halo; and $\alpha=1$ provides a good fit to the data. Reference [7] gave a heuristic argument, pointing out that Eq. (34) relates the soliton scale radius (chosen as the core radius $x_{c}$ in [7]) with the velocity dispersion of particles in the host halo, in qualitative agreement with a wavelike "uncertainty principle."

However, there is another way to express Eq. (34). The core mass of a $\chi_{\lambda}$ soliton is related to its total mass via $M_{c \lambda} \approx 0.236 M_{\lambda}$. Thus, using Eq. (25) we have an analytic relation $M_{c \lambda} \approx 1.02\left(\frac{\left|E_{\lambda}\right|}{M_{\lambda}}\right)^{\frac{1}{2}} \frac{M_{\mathrm{pl}}^{2}}{m}$. This allows us to rephrase the empirical Eq. (34) by a more intuitive (though equally empirical) expression:

$$
\left.\left.\frac{E}{M}\right|_{\text {soliton }} \approx \frac{E}{M}\right|_{\text {halo }} .
$$

Therefore, the soliton-host halo relation in the simulations of Ref. [6,7] can be summarized by the statement that the energy per unit mass of the soliton matches the energy per unit mass of the host halo.

\section{B. Soliton vs host halo: The simulations of Ref. [13]}

The simulations of Ref. [13] pointed to an empirical scaling relation between the soliton mass $M$ and the total energy of the ULDM distribution in the simulation box, $E_{h}$,

$$
\frac{M}{\left(M_{\mathrm{pl}}^{2} / m\right)} \approx 2.6\left|\frac{E_{h}}{\left(M_{\mathrm{pl}}^{2} / m\right)}\right|^{\frac{1}{3}} .
$$

However, this is just Eq. (23), if we replace the halo energy $E_{h}$ by the energy of the soliton. Because the central density profile found in [13] was a $\chi_{\lambda}$ soliton, to a good approximation, it must be the case that the total energy of the halo in the simulations of [13] was dominated by the central soliton contribution. This situation is unlikely to hold for realistic cosmological host halos with $M_{h}$ significantly above $M_{\mathrm{h}, \min }$.

How could this have happened? The initial conditions in the simulations of [13] were a collection of $\mathrm{N}$ solitons, which were then allowed to merge. It appears that these initial conditions were constructed such that one initial state soliton-the soliton of initially largest mass-grew to absorb the entire energy of the system. Differently from Ref. [7] that considered initial conditions of $\mathrm{N}$ identical initial solitons, the simulations of [13] initiated their $\mathrm{N}$ solitons with a random flat distribution in soliton radius. Such distribution would be skewed towards large soliton energy because $E_{\lambda} \propto x_{c \lambda}^{-3}$. Considering the initial condition set-up as explained in [13], we find that the most massive initial state soliton typically needed to grow in mass by only a factor of $1.5 \div 2$, to absorb the entire energy of the halo.

Note that energy dominance of the central soliton over the host halo, implied by Eq. (36), is not the same, of course, as equating the energy per unit mass of the soliton and the halo, implied by Eq. (35). Halos in [6,7] attained masses up to two orders of magnitude larger than the central soliton mass, meaning their halo energy was two orders of magnitude larger than the energy of the soliton. 


\section{Comments}

As far as we can currently determine, Eq. (35) may indeed reflect a realistic soliton-host halo relation for large enough cosmological halos. In the following sections, we take a leap of faith and assume that the simulations of [6,7] produced the correct scaling relation. We stress that Eq. (35) is an empirical result and was only tested in $[6,7]$ for host halo masses ranging from $\sim 10^{8} \mathrm{M}_{\odot}$ to $\sim 10^{11} \mathrm{M}_{\odot}$. Our key numerical analysis will concern systems in this range of mass.

We defer a theoretical study of the origin of Eq. (35) to future work. Here we give only a few comments. We stress that the discussion in the rest of this section does not affect any of our results.

For a soliton, $E / M=\gamma / 3$. On the other hand, $\gamma m$ can be associated with the chemical potential of ULDM particles in the soliton (see e.g., [34] and references therein). This may appear to hint that Eq. (35) corresponds to thermodynamic equilibrium between the ULDM particles in the host halo and in the soliton. However, there is some evidence to the contrary from simulations.

Reference [29] simulated ULDM, adding collisionless point particles ("stars"). The stars aggregated dynamically in a cuspy profile, resulting in a more massive soliton compared to the pure ULDM simulations [6,7] with a given host halo mass. Testing the reversibility of the system, Ref. [29] adiabatically "turned off" the stars after the initial system virialized. When eliminating the stars, the soliton + halo system did not relax back to Eq. (35). Instead, the excess ULDM mass that was contained in the soliton in the presence of stars remained captured in the soliton, and did not return to the host halo. The final state of the system was not described by Eq. (35): the soliton ended up containing larger (negative) $E / M$ than the halo, and larger mass compared with Eq. (31).

\section{SOLITON-HOST HALO RELATION AND GALACTIC ROTATION CURVES}

As we have seen, the soliton-host halo relation found in the simulations of $[6,7]$ can be summarized by Eq. (35), equating the energy per unit mass of the virialized host halo to that in the soliton component. For a virialized system, the energy per unit mass maps to kinetic energy density: in particular, the characteristic circular velocity (or, up to an $\mathcal{O}(1)$ geometrical factor, the velocity dispersion) of test particles in the halo and in the soliton should match. The peak circular velocity of the soliton, given by Eqs. (27)-(28), occurs deep in the inner part, $x<1 \mathrm{kpc}$, of the galaxy; while the peak circular velocity of an NFW-like halo occurs far out at $x \sim 2 R_{s}$, with $R_{s}$ the NFW characteristic radius, of order $10 \mathrm{kpc}$ for a MW-like galaxy. Thus, if the scaling derived from the simulations of $[6,7]$ is correct, ULDM predicts that the peak rotation velocity in the outskirts of a halo should approximately repeat itself in the deep inner region. We now discuss this result quantitatively.
Consider a halo with an NFW density profile

$$
\rho_{\mathrm{NFW}}(x)=\frac{\rho_{c} \delta_{c}}{\frac{x}{R_{s}}\left(1+\frac{x}{R_{s}}\right)^{2}},
$$

where

$$
\rho_{c}(z)=\frac{3 H^{2}(z)}{8 \pi G}, \quad \delta_{c}=\frac{200}{3} \frac{c^{3}}{\ln (1+c)-\frac{c}{1+c}} .
$$

The profile has two parameters: the radius $R_{s}$ and the concentration parameter $c=R_{200} / R_{s}$, where $R_{200}$ is the radius where the average density of the halo equals 200 times the cosmological critical density, roughly indicating the virial radius of the halo. The gravitational potential of the halo is

$$
\Phi_{\mathrm{NFW}}(x)=-\frac{4 \pi G \rho_{c} \delta_{c} R_{s}^{3}}{x} \ln \left(1+\frac{x}{R_{s}}\right) .
$$

Near the origin, $x \ll R_{s}$, $\Phi_{\mathrm{NFW}}$ is approximately constant, $\Phi_{\mathrm{NFW}}\left(x \ll R_{s}\right) \approx \Phi_{h}$, and is related to the mass of the halo, $M_{200}=200 \rho_{c} \frac{4 \pi}{3} c^{3} R_{s}^{3}$, via

$$
\Phi_{h}=-G\left(\frac{4 \pi \delta_{c}}{\left(\ln (1+c)-\frac{c}{1+c}\right)^{2}}\right)^{\frac{1}{3}} \rho_{c}^{\frac{1}{3}} M_{200}^{\frac{2}{3}} .
$$

We can estimate the energy per unit mass of the virialized halo by

$$
\left.\frac{E}{M}\right|_{\text {halo }} \approx \pi \frac{\int_{0}^{R_{200}} d x x^{2} \rho_{\mathrm{NFW}}(x) \Phi_{\mathrm{NFW}}(x)}{M_{200}} .
$$

This gives

$$
\left.\frac{E}{M}\right|_{\text {halo }} \approx \frac{\tilde{c}}{4} \Phi_{h},
$$

where

$$
\tilde{c}=\frac{c-\ln (1+c)}{(1+c) \ln (1+c)-c} .
$$

Typical values of the concentration parameter are in the range $c \sim 5 \div 30$ [35]. In this range, $\tilde{c}$ varies between $\tilde{c} \sim 0.55 \div 0.35$, respectively. (For reference, fits of the MW outer rotation curve give $c \sim 10 \div 20$ [36].)

Plugging Eq. (42) into the soliton-host halo relation Eq. (35), the scaling parameter $\lambda$ is fixed as

$$
-0.23 \lambda^{2} \approx \frac{E_{\lambda}}{M_{\lambda}} \approx \frac{\tilde{c}}{4} \Phi_{h}
$$

which implies ${ }^{5}$

\footnotetext{
${ }^{5}$ In the numerical estimates, we use $H_{0}=70 \mathrm{~km} / \mathrm{s} / \mathrm{Mpc}$ for the present-day Hubble constant.
} 


$$
\begin{aligned}
M_{\lambda} \approx & 2.1 \sqrt{-\tilde{c} \Phi_{h}} \frac{M_{\mathrm{pl}}^{2}}{m} \\
\approx & 2.4 \times 10^{9}\left(\frac{m}{10^{-22} \mathrm{eV}}\right)^{-1} \\
& \times\left(\frac{H(z)}{H_{0}}\right)^{\frac{1}{3}}\left(\frac{M_{200}}{10^{12} \mathrm{M}_{\odot}}\right)^{\frac{1}{3}} f(c) \mathrm{M}_{\odot},
\end{aligned}
$$

with

$$
f(c)=0.54 \sqrt{\left(\frac{c}{1+c}\right) \frac{c-\ln (1+c)}{\left(\ln (1+c)-\frac{c}{1+c}\right)^{2}}} .
$$

Eq. (45) depends weakly on the NFW concentration parameter, via the factor $f(c)$ that varies in the range $0.9 \div 1.1$ for $c=5 \div 30$. It agrees parametrically with the simulation result, Eq. (31) [including the redshift dependence, which we have suppressed in Eq. (31)]. It also agrees quantitatively to about $20 \%$; to see this, we need to account for the slightly different definition of the halo mass $M_{h}$, used in [7], and our $M_{200}$. We do this comparison in App. A.

Consider the rotation velocity curve of an ULDM galaxy satisfying Eq. (35). The NFW rotation curve is given by

$$
\frac{V_{\text {circ, } \mathrm{h}}^{2}(x)}{V_{\text {circh } \mathrm{h}}^{2}\left(R_{s}\right)}=\frac{2(1+\xi) \ln (1+\xi)-2 \xi}{\xi(1+\xi)(\ln (4)-1)}, \quad \xi \equiv \frac{x}{R_{S}} .
$$

This halo rotation curve peaks at $x \approx 2.16 R_{s}$ with a peak value

$$
\max V_{\text {circ, h }} \approx 1.37 \times 10^{5}\left(-\Phi_{h}\right)^{\frac{1}{2}} \mathrm{~km} / \mathrm{s} .
$$

On the other hand, in the inner galaxy $x \ll R_{s}$, the circular velocity due to the soliton peaks to a local maximum of

$$
\max V_{\text {circ }, \lambda} \approx 1.51 \times 10^{5}\left(\frac{\tilde{c}}{0.4}\right)^{\frac{1}{2}}\left(-\Phi_{h}\right)^{\frac{1}{2}} \mathrm{~km} / \mathrm{s},
$$

where we used Eq. (44) to fix $\lambda$ and Eq. (28) to relate it to $\max V_{\text {circ, }, \lambda}$.

As anticipated in the beginning of this section, Eq. (35) predicts approximately equal peak circular velocities for the inner soliton component and for the host halo,

$$
\frac{\max V_{\text {circ }, \lambda}}{\max V_{\text {circ, h }}} \approx 1.1\left(\frac{\tilde{c}}{0.4}\right)^{\frac{1}{2}}
$$

independent of the particle mass $m$, independent of the halo mass $M_{200}$, and only weakly dependent on the details of the halo via the factor $(\tilde{c} / 0.4)^{\frac{1}{2}}$. Equation (49) is plotted in Fig. 3 as function of the concentration parameter.

While $\max V_{\text {circ }, \lambda}$ and the approximate equality Eq. (49) are $m$-independent, the soliton peak velocity occurs in an $m$-dependent location,

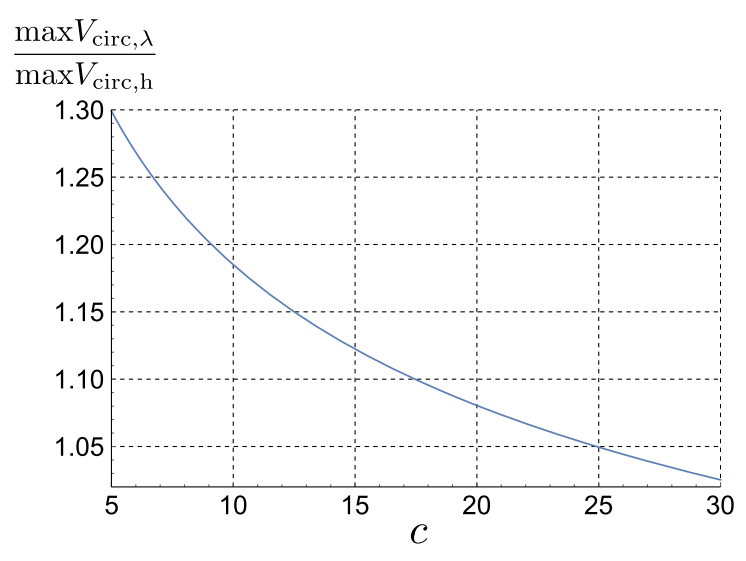

FIG. 3. Ratio between halo and soliton peak circular velocities as a function of the halo concentration.

$$
x_{\text {peak }, \lambda} \approx 184\left(\frac{10^{-22} \mathrm{eV}}{m}\right)\left(\frac{\max V_{\text {circ }, \lambda}}{200 \mathrm{~km} / \mathrm{s}}\right)^{-1} \text { pc. }
$$

Figure 4 shows the circular velocity curve for the NFW halo + soliton system, following from Eq. (35), with ULDM particle mass $m=10^{-22} \mathrm{eV}$. The solid black, dot-dashed orange, and dashed blue lines show the contributions to $V_{\text {circ }}$ due to the total system, the soliton only, and the halo only. Results are shown for three different values of the NFW concentration parameter, $c=10,15$, 25 , with $M_{200}=10^{12} \mathrm{M}_{\odot}$ and $5 \times 10^{10} \mathrm{M}_{\odot}$ on the top and bottom panels, respectively. For larger $m>10^{-22} \mathrm{eV}$, the soliton bump in the rotation curve would shift to smaller $x$ according to Eq. (50), but would maintain its height.

In Fig. 4, to define the rotation velocity for the total system, we set the ULDM mass density for the total system to be $\rho(x)=\max \left\{\rho_{\lambda}(x), \rho_{\mathrm{NFW}}(x)\right\}$, calculate the resulting mass profile $M(x)$, and use spherical symmetry to find $V_{\text {circ }}(x)=\sqrt{G M(x) / x}$. This prescription for matching between the soliton and NFW parts is ad hoc and only roughly consistent with the simulations of $[6,7]$. The true transition region between the NFW part and the soliton part probably deviates from the pure NFW form. Reference [37] considered this transition region and concluded that the density profile in this region should follow approximately $\rho \sim x^{-\frac{5}{3}}$, steeper than the usual inner NFW form $\rho \sim x^{-1}$. This would affect the detailed shape of the rotation curve in the intermediate region between the two peaks, but not our general results.

Equation (49) was derived for an NFW host halo, but it is the manifestation of Eq. (35) that is not tied to a particular parametrization of the halo profile. Building on Eq. (35), we expect in general that for DM-dominated galaxies, the soliton peak circular velocity should roughly equal the peak circular velocity in the host halo. The NFW example demonstrates that details of the host halo profile affect this result at the $10 \%$ level or so. 

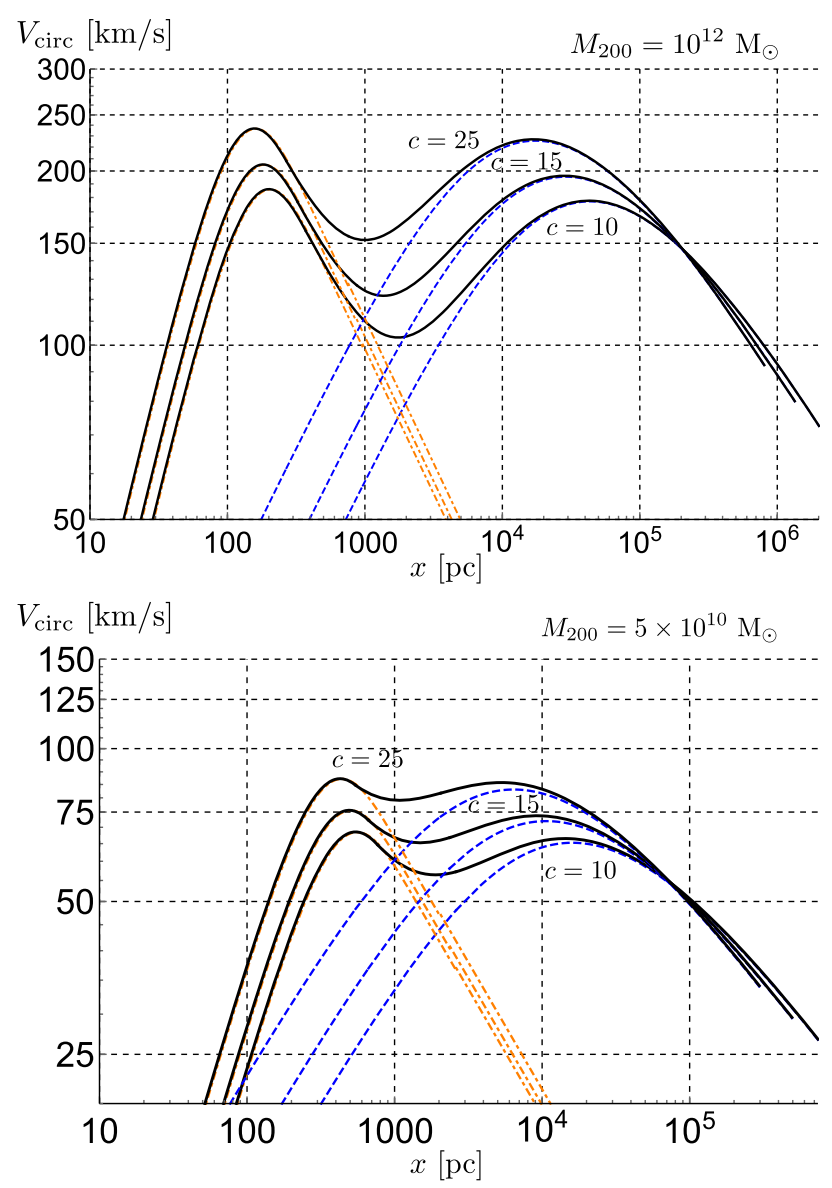

FIG. 4. Rotation curves for the ULDM soliton + halo system, obtained for a DM-only NFW halo using the soliton-halo relation Eq. (35) with $m=10^{-22} \mathrm{eV}$. Solid black, dot-dashed orange, and dashed blue show $V_{\text {circ }}$ due to the total soliton + halo system, the soliton only, and the halo only. Results are shown for NFW concentration parameter $c=10,15$, 25, with $M_{200}=10^{12} \mathrm{M}_{\odot}$ and $5 \times 10^{10} \mathrm{M}_{\odot}$ on the upper and lower panels, respectively.

In the rest of this paper, when we refer to Eq. (49), we set the RHS to unity. Approximating the RHS of Eq. (49) by unity, and replacing max $V_{\text {circ, h }}$ instead of $\max V_{\text {circ }, \lambda}$ in Eq. (28), the peak circular velocity of a host halo allows us to predict the scale parameter $\lambda$ and thus the soliton relevant for that host halo.

\section{A. Comparison to numerical simulations}

In Fig. 5, we compare our results to two soliton + halo configurations from the simulations of [6,29] (for [6], we take the largest halo, and for [29] we take the initial state of Case C). To calculate the soliton, we read $\max V_{\text {circh }}$ from the large-scale peak (at $x \sim 20 \mathrm{kpc}$ ) of the numerically extracted halo rotation curves (solid lines). Following Eq. (49), we use $\max V_{\text {circ,h }}$ instead of $\max V_{\text {circ }, \lambda}$ in Eq. (28), and read off the value of $\lambda$. The predicted soliton bump is shown in dashed lines. It gives the correct soliton peak rotation velocity to $\sim 20 \%$ accuracy in both cases.

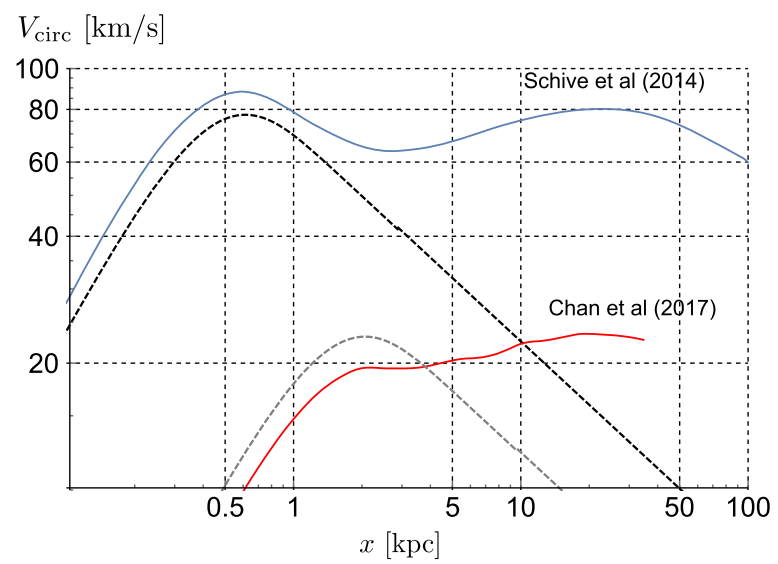

FIG. 5. Comparison of the prediction of Eq. (49) (dashed lines) to the numerical simulation results (solid lines) of Schive et al. (2014) [6] and Chan et al. (2017) [29].

In Fig. 6, we show the velocity profiles for 11 simulated halos, calculated for 6 halos from [7] (solid lines) and 5 halos from [6] (dashed lines). The rotation curves are scaled and normalized such that $x_{\text {peak }, \lambda}=1$ and $\max V_{\text {circ }, \lambda}=1$ in each case. All of the halos satisfy $0.65<\frac{\max V_{\text {circ, } \lambda}}{\max V_{\text {circ, },}}<1.4$; for later reference, the shaded band highlights the range $0.5<\frac{\max V_{\text {circ. },}}{\max V_{\text {circ. }}}<1.5$.

\section{B. Comparison to real galaxies}

We now consider some observational consequences of our analysis. We choose to do so by examining the rotation curves of nearby disc galaxies with halo masses in the range covered by the simulations of [6,7], and above the minimal mass of an ULDM halo, see Eq. (33). We divide our analysis into two parts. First, in Sec. IV B 1, we concentrate

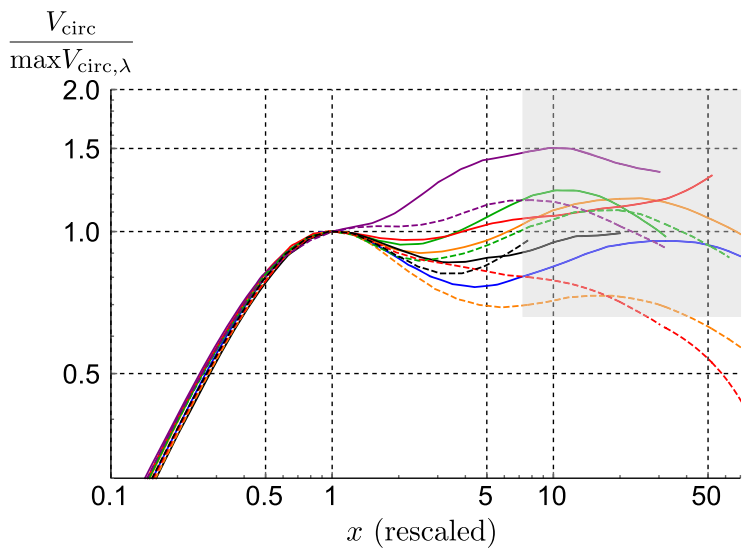

FIG. 6. Circular velocity profiles for halos, calculated for simulation results published in [7] (solid lines) and [6] (dashed lines), scaled and normalized such that $x_{\text {peak }, \lambda}=1$ and $\max V_{\text {circ }, \lambda}=1$. Shaded band highlights the range $0.5<\frac{\max V_{\text {circ, } \lambda}}{\max V_{\text {circ, h }}}<1.5$. 
on a small set of galaxies for which high-resolution kinematical and photometric data are available. For this small set of galaxies, we present detailed rotation curve data and compare them to the ULDM prediction. We find clear tension between the data and the ULDM prediction. Next, in Sec. IV B 2, we extend the analysis to include a large sample of galaxies, confirming and reinforcing the tension between the ULDM prediction and the rotation curve data.

\section{A few specific examples}

In our first analysis of the data, we study a set of four representative rotation curves from Ref. [27] (see Ref. [28] for a recent rendering of these and many other rotation curves), for which high-resolution kinematical data are available. Our goal is to check if the ULDM prediction, that we demonstrated in the previous section using simulated galaxies, actually holds in real observed galaxies. The key prediction we check is summarized by Eq. (49), and was demonstrated for simulated galaxies in Figs. 5 and 6: this prediction states that given a measurement of the largeradius halo rotation curve, ULDM prescribes a solitoninduced peak in the inner part of the halo, with height specified by Eq. (49), at a location specified by Eq. (50).

The four measured rotation curves are shown in Figs. 7-10, as blue markers. For each of these rotation curves, we use the measured circular velocity at the farthest radius, to serve as an input for max $V_{\text {circ, h }}$ in Eq. (49). In turn, Eq. (49) gives as output the predicted soliton-induced peak rotation velocity, $\max V_{\text {circ, } \lambda}$. Given the solitoninduced peak rotation velocity, the soliton $\lambda$ parameter is fixed by Eq. (28) and with it, the full soliton-induced rotation curve. The result is plotted as dashed line in Figs. 7-10; the upper panels show the result for $m=$ $10^{-22} \mathrm{eV}$ and the lower panels show it for $m=10^{-21} \mathrm{eV}$.

We find that the ULDM soliton-host halo relation significantly overestimates the rotation velocity in the inner part of all of the galaxies in Figs. 7-10. This puts the predictions of ULDM in the mass range $m \sim\left(10^{-22} \div\right.$ $\left.10^{-21}\right) \mathrm{eV}$ in tension with the data.

We emphasize that in using Eq. (49) to predict the soliton, we set the RHS of that equation to unity, and thus we ignore any details of the shape of the host halo. We also neglect corrections due to baryons. On the one hand, as we have learned from the NFW analysis, this prescription for deriving the soliton profile would suffer $\mathcal{O}(10 \%)$ corrections from the detailed halo shape. The baryonic (stellar and gas) contribution to the gravitational potential affects the halo velocity at a similar level: in Ref. [28], the baryonic contribution to the halo peak rotation velocity of UGC 1281, UGC 4325, and NGC 100 was estimated from photometric data to be well below the DM contribution, ${ }^{6}$

\footnotetext{
${ }^{6}$ For this estimate, we use $3.6 \mu \mathrm{m}$ mass-to-light ratio $\Upsilon_{*}=$ $0.5 \mathrm{M}_{\odot} / \mathrm{L}_{\odot}$.
}
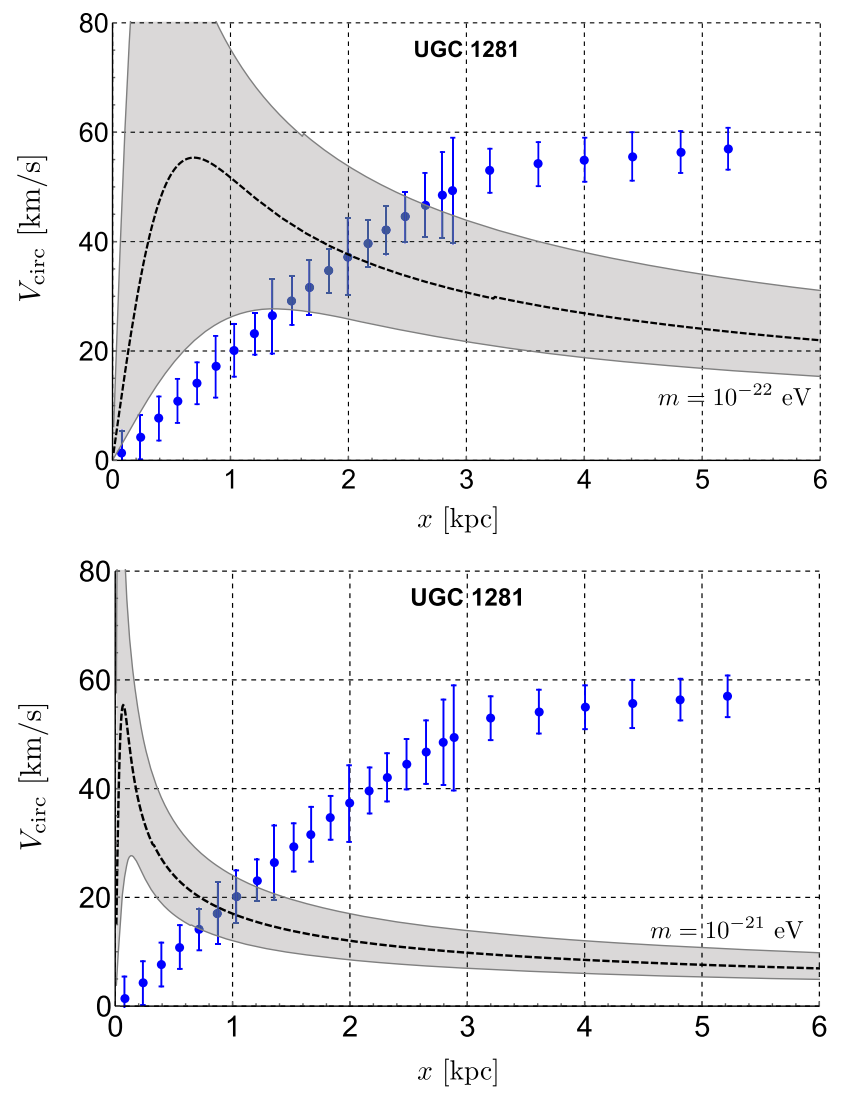

FIG. 7. Measured rotation curve of UGC 1281 superimposed on the prediction from Eq. (49) following from the soliton-host halo relation. The ULDM mass is $m=10^{-22} \mathrm{eV}$ (upper panel) and $m=10^{-21} \mathrm{eV}$ (lower panel). The shaded band accounts for the intrinsic scatter of the soliton-host halo relation.

$V_{\text {circh h }}^{(\mathrm{bar})} / V_{\text {circh h }}^{(\text {obs }}<0.5$. This means that the observed velocity $V_{\text {circh h }}^{\text {(obs }}$ is equal to the DM-induced velocity $V_{\text {circh h }}^{(\mathrm{DM})}$ to better than $15 \%$. On the other hand, this simple procedure relieves us from the need to fit for the virial mass or other details of the host halo. All that is needed is the peak halo rotation velocity, a directly observable quantity. ${ }^{7}$ For completeness, we will return to the issue of baryonic effects and deal with it more systematically in the second part of this section, considering SPARC data [28].

Equation (49) (represented by the dashed line in Figs. 7-10) corresponds to the central value of the soliton-host halo relation. Reference [6,7] showed a scatter of about a factor of two around Eq. (34) between simulated halos. This translates to a factor of two scatter in the soliton $\lambda$ parameter (we have illustrated this scatter for a sub-sample of simulated halos in Fig. 6). In Figs. 7-10, we represent this

\footnotetext{
${ }^{7}$ The rotation curves in Figs. 7-10 do not show a clear peak within the range of the measurement; this means that our soliton bump, derived from the maximal velocity seen in the data, underestimates the true predicted soliton and is thus conservative.
} 

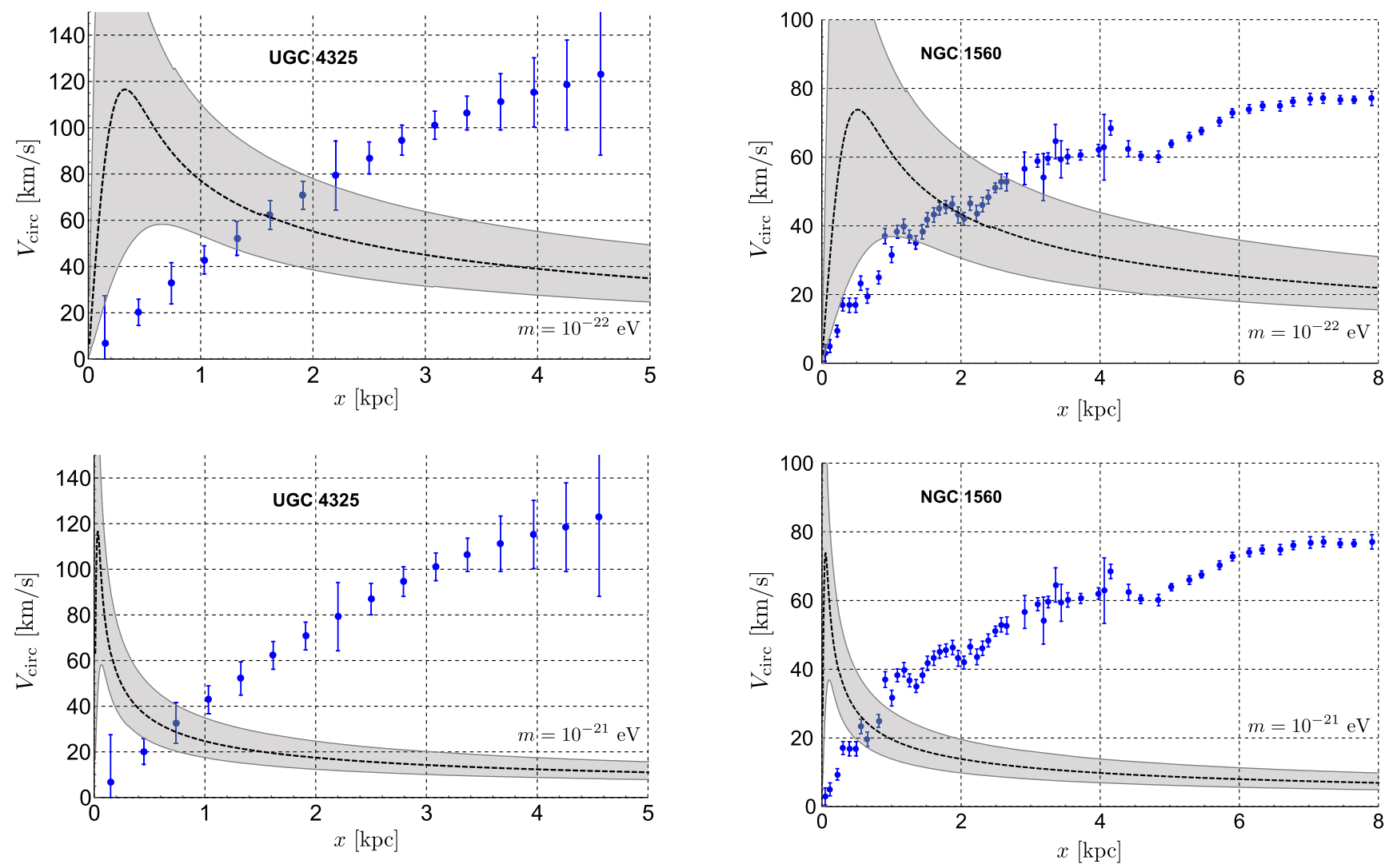

FIG. 8. Same as Fig. 7 for UGC 4325.

scatter by a shaded band, showing the results when the $\lambda$ parameter inferred from Eq. (49) is changed by a factor of 2.

\section{A large sample of galaxies: SPARC}

It is important to check if scatter between different galaxies could explain the discrepancy, with the four galaxies in Figs. 7-10 being accidental outliers. To address this question, we analyze the 175 rotation curves contained in the SPARC data base [28]. This sample includes, in particular, the galaxies UGC 1281, UGC 4325, and NGC 100, shown in Figs. 7-10.

Our SPARC analysis is as follows. For each galaxy, we make a crude estimate of the halo mass contained within the observed rotation curve profile by $M_{\text {gal }} \sim R V^{2} / G$, where $R$ is the radial distance of the last data point in the rotation curve, and $V$ the corresponding velocity. We keep only galaxies with $5 \times 10^{11} \mathrm{M}_{\odot}>M_{\text {gal }}>5 \times 10^{8}\left(\mathrm{~m} / 10^{-22} \mathrm{eV}\right)^{-3 / 2} \mathrm{M}_{\odot}$. We do this in order to limit ourselves to galaxy masses that are comfortably above the minimal halo mass (33), and not above the range simulated in [6,7]. Our results are not sensitive to the details of this mass cut.

Next, for each galaxy we determine the observed maximal halo rotation velocity $\max V_{\text {circ,h }}$, and use it to compute the soliton prediction from Eq. (49). To avoid confusion between halo peak velocity and soliton peak velocity, we search for the halo peak velocity restricting to radial distance $x>3\left(\mathrm{~m} / 10^{-22} \mathrm{eV}\right)^{-1} \mathrm{kpc}$. Galaxies with no data above $x=3\left(\mathrm{~m} / 10^{-22} \mathrm{eV}\right)^{-1} \mathrm{kpc}$ are discarded. Our results are not sensitive to this criterion; defining the halo cut anywhere at $\gtrsim 1\left(\mathrm{~m} / 10^{-22} \mathrm{eV}\right)^{-1} \mathrm{kpc}$ guarantees that such confusion is avoided.

SPARC galaxies come with photometric data, allowing us to model the baryonic contribution to the gravitational potential [28]. We use this information to limit baryonic effects on our analysis, and to explore the sensitivity of our results to baryonic corrections. For each galaxy, we estimate the baryonic contribution to the observed rotation velocity using the mass models of [28] with $3.6 \mu \mathrm{m}$ mass-tolight ratio $\Upsilon_{*}=0.5 \mathrm{M}_{\odot} / \mathrm{L}_{\odot}$. Setting $V_{\text {circ,h }}^{(\text {bar }, 2}+V_{\text {circ,h }}^{(\mathrm{DM}), 2}=$ $V_{\text {circ,h }}^{(\text {obs }, 2}$, we calculate the ratio $f_{\text {bar2DM }}=V_{\text {circh },}^{(\mathrm{bar}} / V_{\text {circh } \mathrm{h}}^{(\mathrm{DM})}$. We present results when cutting on different values of $f_{\text {bar2DM }}<1,0.5,0.33$.

Our first pass on the data includes only galaxies for which the predicted soliton is resolved, namely, $x_{\text {peak }, \lambda}$ from Eq. (50), with $\max V_{\text {circ, } \lambda}=\max V_{\text {circh h }}^{(\mathrm{DM})}$, lies within the rotation curve data. For these galaxies, we compute from data the ratio

$$
\frac{V_{\text {circ,obs }}\left(x_{\text {peak }, \lambda}\right)}{\max V_{\text {circh }}^{(\mathrm{DM})}} .
$$



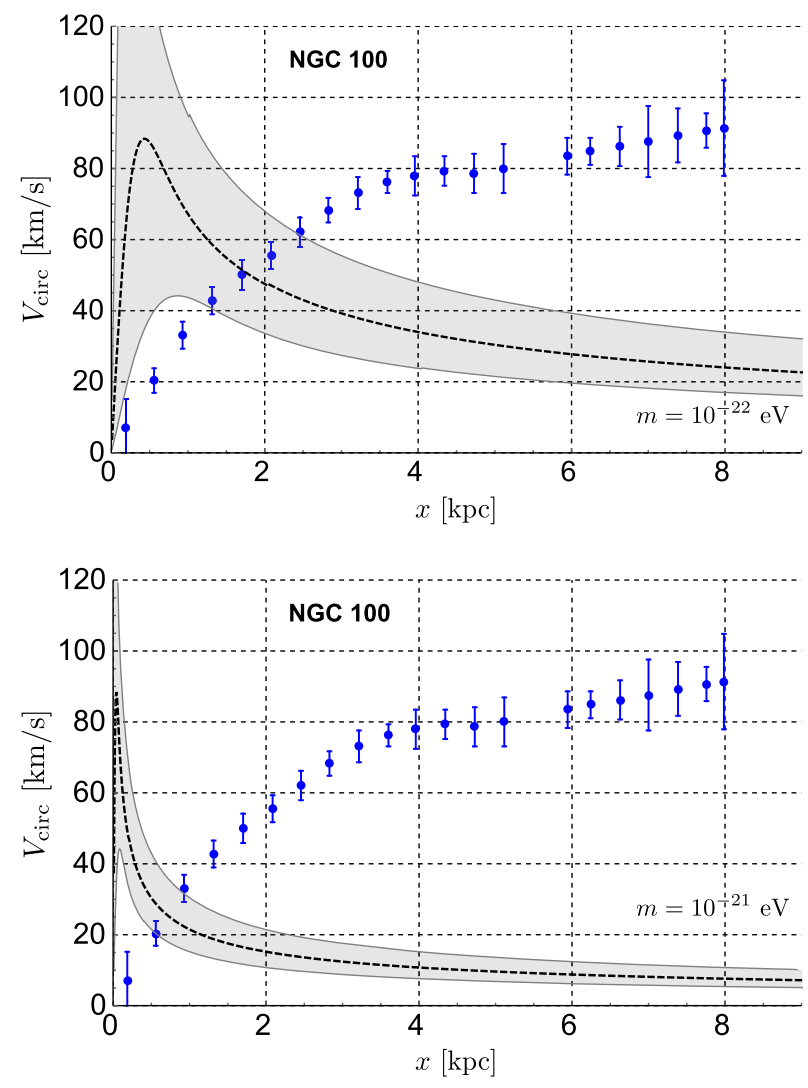

FIG. 10. Same as Fig. 7 for NGC 100.

Here, $V_{\text {circ,obs }}\left(x_{\text {peak }, \lambda}\right)$ is the measured velocity at the expected soliton peak position.

The results of this first pass on the data are shown in Fig. 11. Red, blue, and green histograms show the result when imposing $f_{\text {bar2DM }}<1,0.5,0.33$, respectively. For $m=10^{-22} \mathrm{eV}$, we find 45,26 , and 5 galaxies that pass the resolved soliton cut for $f_{\text {bar2DM }}<1,0.5,0.33$. For $m=10^{-21} \mathrm{eV}$, only 4 galaxies pass the resolved soliton cut for $f_{\text {bar2DM }}<1$, and none for $f_{\text {bar2DM }}<0.5,0.33$.

Including only galaxies with a resolved soliton causes us to lose many rotation curves with discriminatory power. For example, UGC 4325 drops out of the analysis for $m=10^{-21} \mathrm{eV}$, though it clearly constrains the model, as seen from the lower panel of Fig. 8. To overcome this without complicating the analysis, we perform a second pass on the data. Here, we allow galaxies with unresolved soliton, as long as the innermost data point is located not farther than $3 \times x_{\text {peak }, \lambda}$. We need to correct for the fact that the soliton peak velocity is outside of the measurement resolution. To do this, we modify our observable as

$$
\frac{V_{\text {circ,obs }}\left(x_{\text {peak }, \lambda}\right)}{\max V_{\text {circh }}^{(\mathrm{DM})}} \rightarrow \frac{V_{\text {circ,obs }}\left(x_{\text {min,data }}\right)}{\max V_{\text {circ, h }}^{(\mathrm{DM})}} \times \sqrt{\frac{x_{\text {min,data }}}{x_{\text {peak }, \lambda}}},
$$

where $x_{\text {min,data }}$ is the radius of the first data point. This correction is conservative, because it takes the fall-off of the
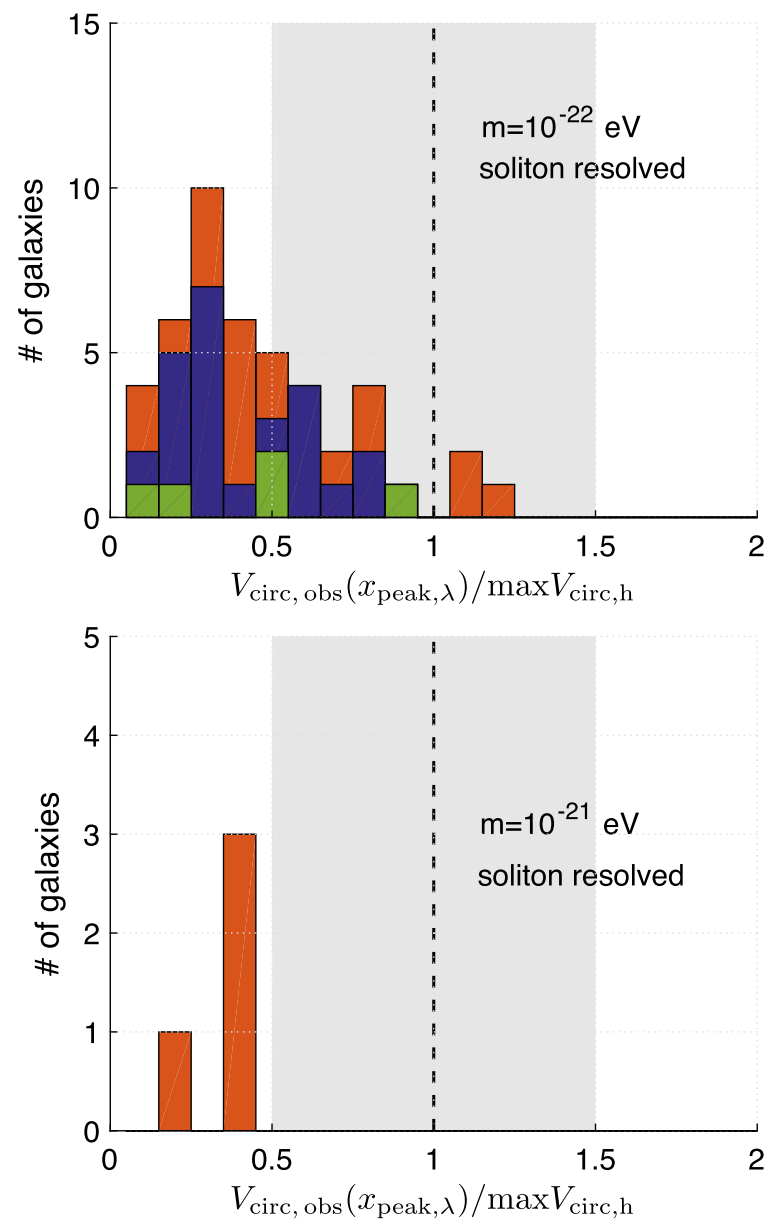

FIG. 11. Distribution of SPARC galaxies [28] with respect to the ratio of observed circular velocity at the soliton peak to the maximal circular velocity of the halo. The vertical dashed line shows the prediction for the mean implied by the soliton-host halo relation and the shaded region accounts for the intrinsic scatter in this relation. The ULDM mass is $m=10^{-22} \mathrm{eV}$ (upper panel) and $m=10^{-21} \mathrm{eV}$ (lower panel). Red, blue, green histograms correspond to the cuts $f_{\text {bar2DM }}<1,0.5,0.33$, respectively. Only rotation curves with resolved solitons are included (see the main text for details).

soliton gravitational potential at $x>x_{\mathrm{peak}, \lambda}$ to be the same as for a point mass. In reality, the potential decays slower and the soliton-induced velocity decreases slower. Keeping this caveat in mind, Fig. 12 presents our results including unresolved solitons. For $m=10^{-22} \mathrm{eV}$, with $f_{\text {bar2DM }}<1,0.5$, we find 48 and 16 galaxies with unresolved soliton, that can be added to the sample of Fig. 11. No galaxy is added for $f_{\text {bar2DM }}<0.33$. For $m=10^{-21} \mathrm{eV}$, 16 and 5 galaxies are added with $f_{\text {bar2DM }}<1,0.5$, and none for $f_{\text {bar2DM }}<0.33$.

In Figs. 11-12, vertical dashed line indicates the solitonhost halo prediction. The shaded region shows the range of the prediction, modifying the RHS of Eq. (49) between $0.5-1.5$, consistent with the scatter seen in the simulations. 

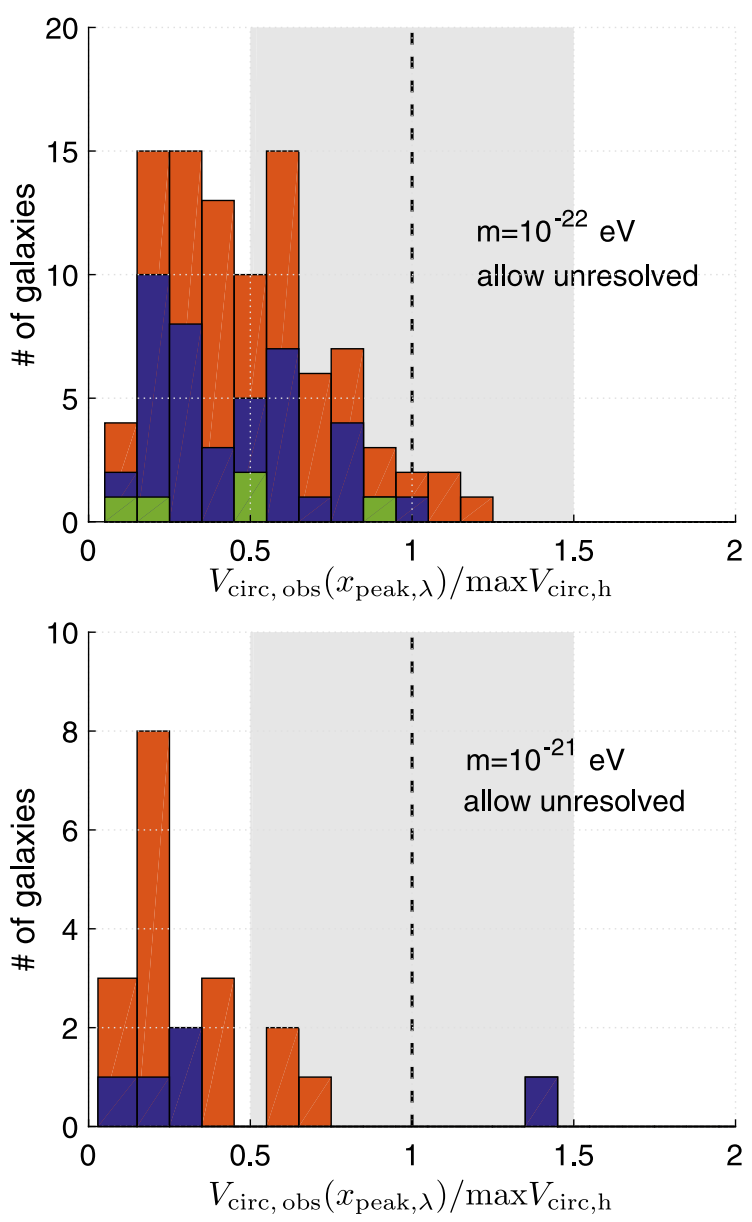

FIG. 12. Same as Fig. 11, including galaxies with unresolved solitons (see the main text).

We conclude that the four galaxies in Figs. 7-10 are not outliers: they are representative of a systematic discrepancy, that would be difficult to attribute to the scatter seen in the simulations. If the soliton-host halo relation of $[6,7]$ is correct, then ULDM in the mass range $m \sim 10^{-22} \mathrm{eV}$ to $m \sim 10^{-21} \mathrm{eV}$ is in tension with the data.

We have limited our attention to the range $m=\left(10^{-22} \div 10^{-21}\right) \mathrm{eV}$, for which we believe the results are clear. We leave a detailed study of the precise exclusion range to future work. We note that for lower particle mass, $m \lesssim 10^{-23} \mathrm{eV}$, the soliton contribution extends over much of the velocity profile of many of the SPARC galaxies, leaving little room for a host halo. This limit, where the galaxies are essentially composed of a single giant soliton, was considered in other works. We do not pursue it further, one reason being that this range of small $m$ is in significant tension with Ly- $\alpha$ data $[18,19]$.

For higher particle mass, $m \gtrsim 10^{-21} \mathrm{eV}$, the soliton peak is pushed deep into the inner $100 \mathrm{pc}$ of the rotation curve. Although high-resolution data (e.g., NGC 1560, Fig. 9) are sensitive to and disfavors this situation, a more careful analysis would be needed to draw a definitive conclusion. Note that in this range of $m$, ULDM ceases to offer a solution to the small-scale puzzles of $\Lambda \mathrm{CDM}$ (see, e.g., review in [12]).

\section{BARYONIC EFFECTS}

\section{A. A fixed distribution of baryons}

We now consider the soliton solution, and some aspects of the soliton-host halo relation, with a coexisting distribution of baryonic mass. In this section, we consider a smooth mass distribution, deferring the analysis of the effect of a super-massive black hole (SMBH) to App. B.

The analysis of baryonic effects is qualitatively important, and quantitatively relevant to ULDM in, e.g., MW-like galaxies. We will see that in order to make a significant impact, baryons need to constitute an $\mathcal{O}(1)$ fraction of the total mass in the soliton region. For many of the galaxies that we analyzed in Sec. IV B, the predicted soliton contribution to the rotation velocity exceeds the observed velocities by a factor of 3-5, implying that the soliton overpredicts the mass in the central region of the galaxy by about an order of magnitude. This leaves little room for baryons (and, we think, baryonic feedback) to significantly affect the dynamics of the inner ULDM halo.

Proceeding to the analysis, we denote the gravitational potential due to baryons by $\Phi_{b}(r)$. We assume that the distribution of baryonic mass is spherically symmetric $^{8}$ and dies off at infinity sufficiently fast, so that $\Phi_{b}(r \rightarrow \infty)=-G M_{b} / r$, where $M_{b}$ is the total baryonic mass. Adding $\Phi_{b}(r)$ changes Eqs. (7)-(8) into [2]

$$
\begin{gathered}
\partial_{r}^{2}(r \chi)=2 r\left(\Phi+\Phi_{b}(r)-\gamma\right) \chi, \\
\partial_{r}^{2}(r \Phi)=r \chi^{2} .
\end{gathered}
$$

It remains convenient to solve the problem using boundary conditions with $\chi(0)=1$. Let us denote this solution (satisfying $\chi(0)=1$ ) by $\chi_{1}\left(r ; \Phi_{b}\right)$, accompanied by the soliton potential $\Phi_{1}\left(r ; \Phi_{b}\right)$. The general solution $\chi_{\lambda}\left(r ; \Phi_{b}\right)$ satisfying Eqs. (53)-(54) with boundary condition $\chi_{\lambda}\left(0 ; \Phi_{b}\right)=\lambda^{2}$ is given by

$$
\begin{gathered}
\chi_{\lambda}\left(r ; \Phi_{b}\right)=\lambda^{2} \chi_{1}\left(\lambda r ; \lambda^{-2} \Phi_{b}\left(\lambda^{-1} r\right)\right), \\
\Phi_{\lambda}\left(r ; \Phi_{b}\right)=\lambda^{2} \Phi_{1}\left(\lambda r ; \lambda^{-2} \Phi_{b}\left(\lambda^{-1} r\right)\right) .
\end{gathered}
$$

Defining the soliton mass and energy in the presence of the baryons by $M_{\lambda}\left(\Phi_{b}\right), E_{\lambda}\left(\Phi_{b}\right)$, we have

$$
M_{\lambda}\left(\Phi_{b}\right)=\lambda M_{1}\left(\lambda^{-2} \Phi_{b}\left(\lambda^{-1} r\right)\right)
$$

\footnotetext{
${ }^{8}$ The assumption of spherical symmetry is not realistic; some of the galaxies considered in Sec. IV B may in fact exhibit maximal discs. Nevertheless, we expect our simplified analysis to give us correct order of magnitude estimates and defer the analysis of nonspherical baryonic + soliton + halo systems to future work.
} 


$$
E_{\lambda}\left(\Phi_{b}\right)=\lambda^{3} E_{1}\left(\lambda^{-2} \Phi_{b}\left(\lambda^{-1} r\right)\right)
$$

where the ULDM energy is

$$
E\left(\Phi_{b}\right)=\int d^{3} x\left(\frac{|\nabla \psi|^{2}}{2 m^{2}}+\left(\frac{\Phi}{2}+\Phi_{b}(x)\right)|\psi|^{2}\right) .
$$

Given the function $\Phi_{b}$, and plotting $M_{\lambda}\left(\Phi_{b}\right)$ and $E_{\lambda}\left(\Phi_{b}\right)$ vs $\lambda$, we can find the value of $\lambda$ and hence the profile for a soliton solution of any desired mass or energy.

Solutions of Eqs. (55)-(56) can be compared with the results of numerical simulations. Reference [29] added a distribution of mass in the form of point particles ("stars") to ULDM simulations with $m=0.8 \times 10^{-22} \mathrm{eV}$. The first toy model they studied included an isolated soliton (without an ULDM host halo) of mass $M=3.3 \times 10^{8} \mathrm{M}_{\odot}$, to which a stellar distribution was added and evolved to a virialized state. From Fig. 1 of Ref. [29], we derive the
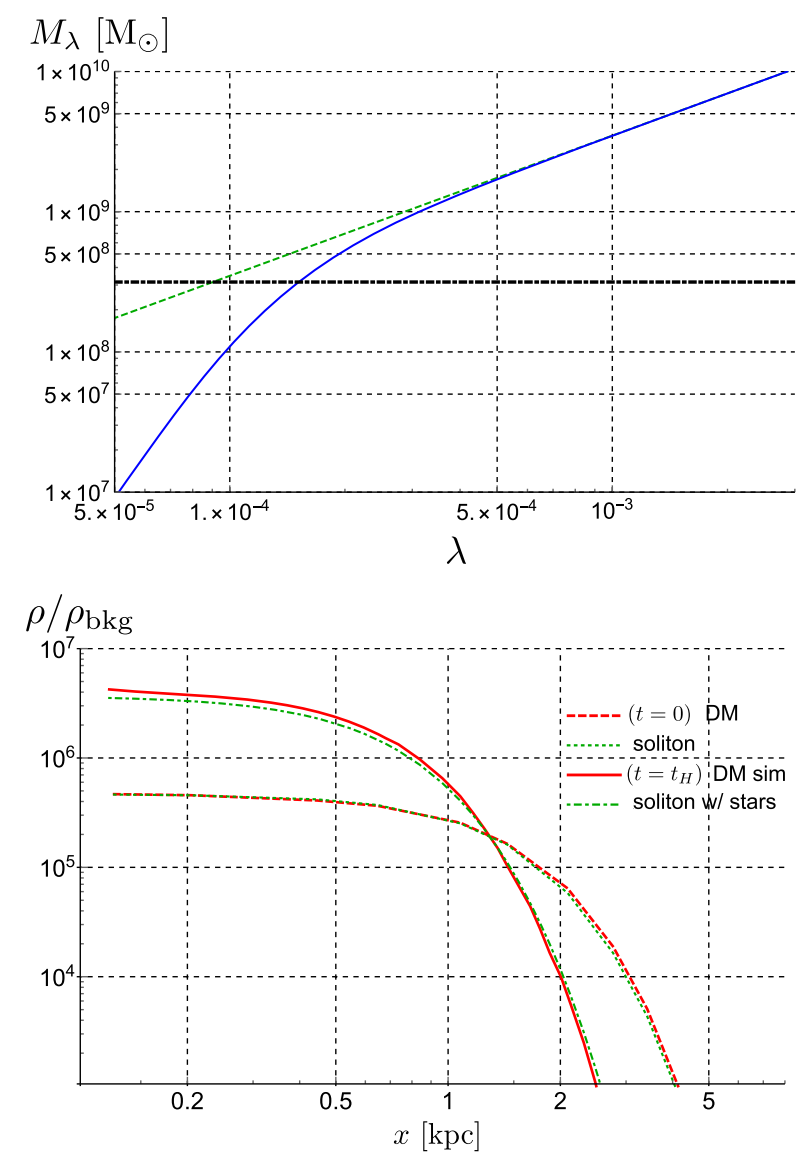

FIG. 13. Top: Soliton mass vs $\lambda$ parameter, determined using Eq. (57) with the stellar gravitational potential as in the numerical simulation shown in Fig. 1 of Ref. [29]. Blue solid (green dashed) lines show the $M_{\lambda}$ vs $\lambda$ with (without) the stellar potential. Black horizontal (dot-dashed) line denotes the mass $M$ chosen in the simulation. Bottom: Soliton profiles with and without stars, describing the results of numerical simulation shown in Fig. 1 of Ref. [29] ( $\rho_{\text {bkg }}$ is the cosmological background matter density). baryonic contribution to the gravitational potential, and solve for the distorted soliton at the stated ULDM mass. In the top panel of Fig. 13, we show $M_{\lambda}$ vs $\lambda$, derived from Eq. (57). Blue solid (green dashed) lines show $M_{\lambda}$ with (without) the stellar potential. Black horizontal line denotes the value of $M$ that was fixed in the simulation: the intersection of the black and the blue lines gives the parameter $\lambda$ describing the distorted soliton. In the bottom panel, we show the density profiles. Red lines are taken from [29], while green lines show the analytic soliton solutions. Clearly, the numerical profile from [29] closely matches the analytically-derived distorted soliton of Eq. (55).

Reference [29] also simulated the soliton in an ULDM halo, the output of a DM-only cosmological simulation. Then, a stellar mass distribution was introduced as before and the system allowed to evolve.

When embedded in a halo, the mass of the soliton is not constant anymore but can grow by absorbing mass from the halo. However, regardless of the large-scale halo, in the core region the perturbed soliton profile is still fixed by the SP equations up to the ambiguity of $\lambda$. We checked that in all of the virialized soliton + halo + stellar mass simulations, presented in Ref. [29], the soliton profile matches that of the analytic distorted soliton, once accounting for the stellar potential. In the top panel of Fig. 14, we illustrate this point for the $t=t_{H}$ snapshot of Case C, described in Fig. 5 of Ref. [29].

With stars included, the total $t=t_{H}$ mass distribution, given by summing the red (DM) and green (stars) solid lines in the upper panel of Fig. 14, leads to the rotation curve shown by the red solid line in the lower panel of the same figure. This rotation curve is peaked at small radius due to the distorted soliton, that is more massive than the soliton-halo prediction of the DM-only simulations. The inner velocity exceeds the prediction of Eq. (49) by a factor of two. We also show, by green dashed line, the rotation velocity at $t=t_{H}$ due to ULDM alone. To compare, the blue dotted curve shows the rotation curve of the initial ULDM system, extracted from the cosmological simulations. This is the same curve we showed in Fig. 5; as we discussed, it satisfies Eq. (49) to $20 \%$.

These numerical results suggest that the presence of baryonic matter tends to make the soliton somewhat more compact and massive than predicted by the pure ULDM soliton-host halo relation (31). This, in turn, increases the peak in the rotation curve due to the soliton, suggesting that our constraints on ULDM obtained in Sec. IV B are robust with respect to inclusion of baryons. We now consider an example, where baryonic effects are expected to be important: the Milky Way.

\section{B. The Milky Way: Nuclear bulge vs soliton}

References [6,7] commented that for a MW-like galaxy, Eq. (31) predicts that ULDM with $m \sim 10^{-22} \mathrm{eV}$ should 

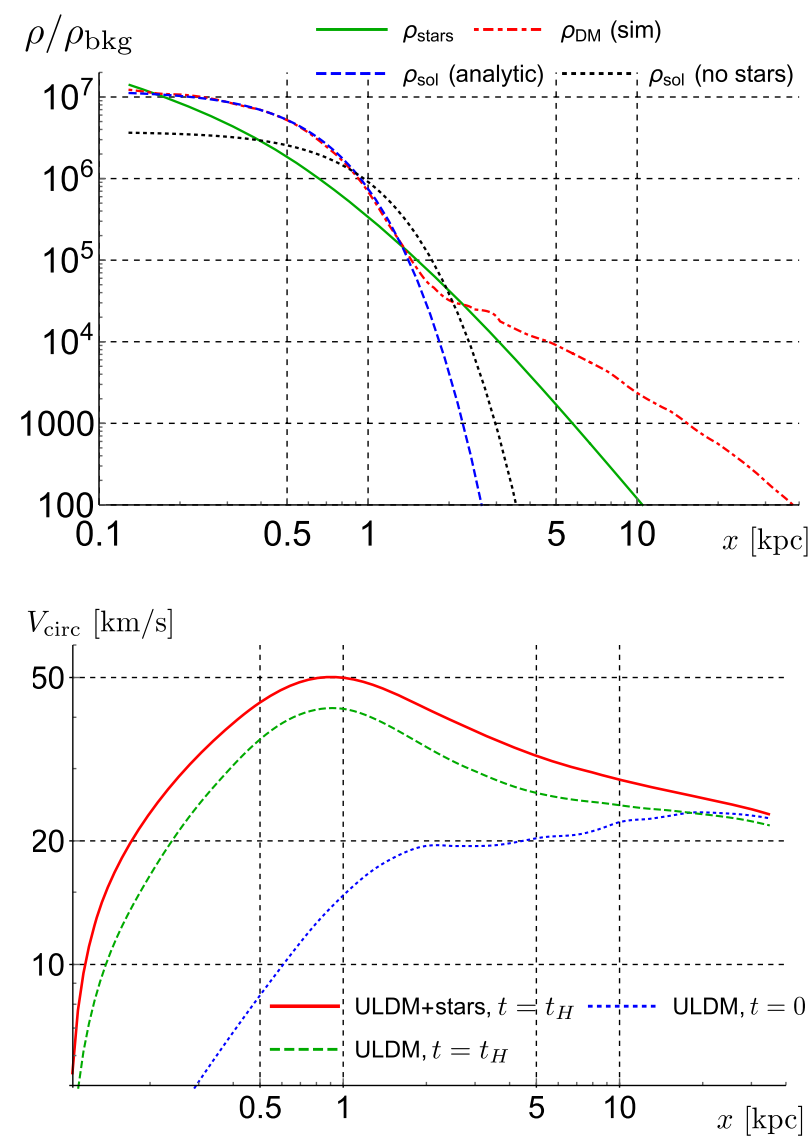

FIG. 14. Top: Soliton profiles with and without stars, describing the inner part of the halo obtained in the numerical simulation (Case C in Fig. 5 of Ref. [29]). Green and red solid lines show the stellar and ULDM densities, respectively, taken from the simulation. Blue dashed show a distorted soliton solution, computed including the stellar potential. Dotted black shows an un-distorted soliton with the same mass. Bottom: Rotation curve for the total ULDM + stellar system (red solid) and including only the ULDM contribution (green dashed), at $t=t_{H}$. We also show the initial ULDM only distribution at $t=0$ (blue dotted).

produce a bump in the rotation curve at a radial distance $\sim 200 \mathrm{pc}$, consistent at face value with a feature observed in the MW. Our results in Sec. IV B show that this value of $m$ is in tension with observations of small disc galaxies. Nevertheless, the MW provides an interesting example for studying the impact of baryons and of a SMBH on an ULDM soliton, and developing intuition as to what extent this effect can be important. Our goal in this section is to provide a preliminary study along these lines, using photometric baryonic mass estimates. As an interesting outcome, we find that precision kinematical studies of the MW inner bulge could in principle be sensitive to ULDM with $10^{-21} \mathrm{eV} \lesssim m \lesssim 10^{-19} \mathrm{eV}$, where the analysis along the lines of Sec. IV B may become challenging.

Figure 15 shows a spherically averaged enclosed mass profile, derived for the MW via various dynamical tracers [38-51]. Flattening of the enclosed mass at small radii

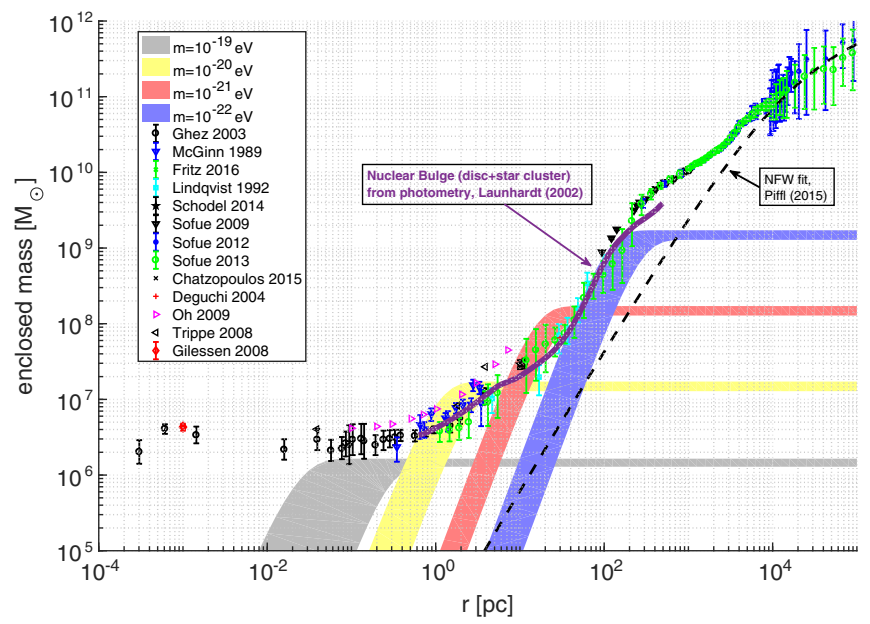

FIG. 15. Spherically averaged mass profile in the Milky Way, vs ULDM soliton contributions. See text for details.

reflects the contribution of the SMBH. From the large radius $(r \gtrsim 10 \mathrm{kpc}$ ) part of the rotation curve, the parameters of the large-scale DM host halo can be extracted by standard analysis. We demonstrate this in Fig. 15 by a black dashed line, showing an NFW profile fitted in Ref. [36] to $r \gtrsim 10 \mathrm{kpc}$ SDSS data. The large-scale analysis yields for the MW an host halo mass roughly in the range $M_{h}=(0.8 \div 2) \times 10^{12} \mathrm{M}_{\odot}$.

Given the host halo mass $M_{h}$, the soliton-host halo relation, Eq. (31), predicts the soliton mass $M$. This, in turn, allows us to extract the soliton scaling parameter $\lambda$ (from Eq. (17), with $M_{\lambda}=M$ ) and with it the entire predicted soliton contribution to the enclosed mass. In Fig. 15, shaded bands show the resulting soliton-induced rotation curves for $m$ in the range $\left(10^{-22} \div 10^{-19}\right) \mathrm{eV}$. The width of the shaded bands comes from varying the host halo mass $M_{h}$ in the range $(0.8 \div 2) \times 10^{12} \mathrm{M}_{\odot}$. The approximate effects of baryon mass contributions, which cannot be neglected in the inner MW and which distort the soliton profile, are included and discussed below.

We stress that the purpose of Fig. 15 is to illustrate the possible signature of ULDM in the inner MW, and not for statistical analyses of the MW mass distribution. Modeling the inner MW is a complicated task. The measurement of inner kinematics of the galaxy, below a few kpc, is subject to large systematic uncertainties due, among other issues, to the effects of the Galactic bar and spiral arm structures [51], which impact tangent-point velocity measurements like those utilized in [50,52]. Our simplified derivation of the spherically averaged mass profile in Fig. 15 combines many tracers with different systematics, and accounts for none of these subtleties.

Reference [53] analyzed the MW central gravitational potential using a large set of observational constraints. In addition to the classical bulge and disc, Ref. [53] found dynamical evidence for the presence of a mass component of $\sim 2 \times 10^{9} \mathrm{M}_{\odot}$ extending to $\sim 250 \mathrm{pc}$. This mass 
component is visible as a mass bump in Fig. 15 (see, e.g., green data points extracted from [50]). Consistent with comments in [6,7], the bump is in tantalizing agreement with the soliton prediction of Eq. (31) for $m=10^{-22} \mathrm{eV}$ (blue shaded band).

Unfortunately, there are about a billion stars in there, too: the bump in the mass profile at $r \sim 200 \mathrm{pc}$ has been associated in the literature with the nuclear bulge (NB). Reference [54] fitted the NB mass and light by a dense disc of stars, with mass density $\rho_{*} \sim 200 \mathrm{M}_{\odot} / \mathrm{pc}^{3}$, scale height $\lesssim 45 \mathrm{pc}$ and scale radius $\sim 230 \mathrm{pc}$. In all, the NB is thought to contain $(1.4 \pm 0.6) \times 10^{9} \mathrm{M}_{\odot}$ in stars, roughly enough to match the dynamically inferred mass. Subsequent kinematic detection supporting the stellar mass and disclike morphology of this component was given in [55]. Microlensing analyses [56] lend further support to the results of [53-55] down to $r \gtrsim 220 \mathrm{pc}$.

The photometrically-derived NB mass model of [54] is superimposed as purple line in Fig. 15. We stress that the photometric derivation is subject to large uncertainties due to the need to correct for very strong extinction and due to unknown stellar mass-to-light ratios. What we learn from this photometric mass model, therefore, is that stars could plausibly account for all of the kinematically inferred mass in this region.

Assuming that the NB is due to stars, we now use a toy model of this mass distribution to see its effect on an ULDM soliton. We replace the disclike morphology of the NB in [54] by a spherical model with the same radially averaged mass. The nominal model, containing the NB and additional subleading components described in [54], contains $\sim 1.7 \times 10^{9} \mathrm{M}_{\odot}$ in stars inside of $r=300 \mathrm{pc}$. Adding a $\mathrm{SMBH}$ of $M_{\mathrm{BH}}=4.3 \times 10^{6} \mathrm{M}_{\odot}$ [38], we calculate soliton solutions in this baryonic potential.

Figure 16 shows the soliton mass as function of the $\lambda$ parameter, for $m=10^{-22} \mathrm{eV}$. Green dashed line shows the unperturbed $M_{\lambda}$ vs $\lambda$ relation. Solid, dashed, and dotted

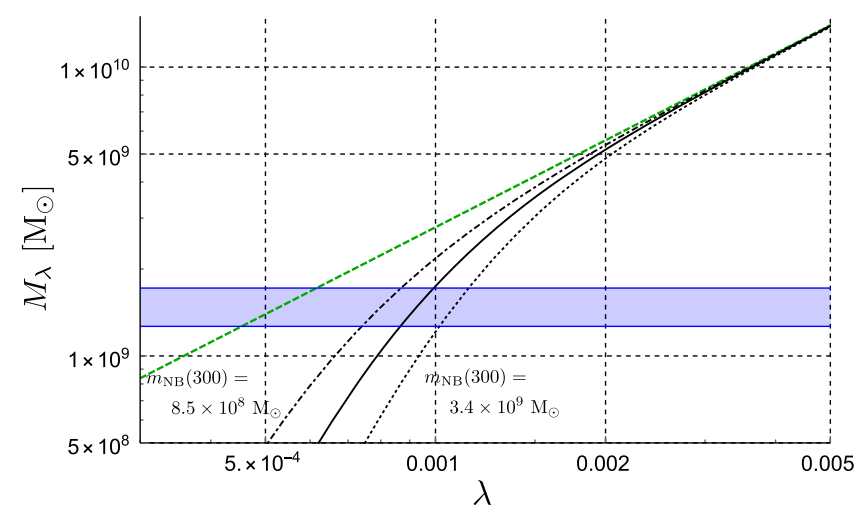

FIG. 16. Soliton mass vs $\lambda$ parameter, accounting for the spherically averaged gravitational potential due to stars [54]. The ULDM particle mass is $m=10^{-22} \mathrm{eV}$. See text for more details. black lines show the relation for the nominal NB model and for two other models, obtained by scaling the NB mass density by an over-all factor of 0.5 and 2 , respectively. For orientation, shaded blue band shows the soliton mass predicted by Eq. (31) for a host halo with mass $M_{h}=(0.8-2) \times 10^{12} \mathrm{M}_{\odot}$.

For $M_{\lambda} \gtrsim 3 M_{\mathrm{NB}} \sim 5 \times 10^{9} \mathrm{M}_{\odot}$, the NB makes a negligible impact on the soliton. For larger ratio of the stellar to ULDM mass, $M_{\lambda} \lesssim M_{\mathrm{NB}}$, the NB becomes important, contracting the soliton profile. For the MW, this is the parametric region predicted by Eq. (31), implying that the solitons would receive significant distortion. In Fig. 15, we illustrated this effect by presenting, in shaded bands, the soliton mass profiles computed accounting for the nominal NB model. We observe that the solitons for $m$ in the range $\left(10^{-22} \div 10^{-19}\right)$ eV are expected to affect the potential at an order unity level. Thus, a dedicated analysis using our formalism to calculate steady state soliton profiles consistent with a given baryonic mass model could be sensitive to ULDM solitons all the way up to $m \sim 10^{-19} \mathrm{eV}$. For larger $m$, the expected soliton becomes subdominant with respect to the $\mathrm{SMBH}$.

Another limitation comes from absorption of the ULDM from the soliton by SMBH. As discusses in App. B 2, the accretion rate is negligible as long as $m \lesssim 5 \times 10^{-20} \mathrm{eV}$. However, for higher ULDM masses the time scale of accretion can become shorter than the age of the universe and our steady-state Newtonian analysis in this section may not apply.

\section{DISCUSSION}

\section{A. Comparison to previous work}

An earlier analysis of ULDM halos including the effect of baryons was done in [2] (see also [3]). This work attempted to fit the rotation curves of spiral galaxies from [57], assuming that the entire ULDM halo is contained in a giant soliton (see also [58] for a similar approach in modeling low surface brightness galaxies). This exercise led [2] to report $m<10^{-23} \mathrm{eV}$, in tension with Ly- $\alpha$ data. This exercise is different from the current work. As suggested by numerical simulations, we expect heavier ULDM particles with $m \gtrsim 10^{-22} \mathrm{eV}$ to produce galaxies with a large scale host halo following roughly the usual NFW profile, in which the soliton affects the rotation curve only in the inner part of the halo.

References $[6,8]$ performed a Jeans analysis, fitting a soliton + halo configuration to a small sample of dispersion-dominated MW-satellite dwarf spheroidal galaxies (dSph). They found that the modeling of stellar kinematics in the $\mathrm{dSph}$ is consistent with a cored inner region, as expected in ULDM, with good fits to the data for $m \lesssim 10^{-22} \mathrm{eV}$. The fitted core found in [6] was consistent with expectations from the soliton-host halo relation. In [8], the transition between an assumed host halo NFW 
profile and the soliton was modeled using corresponding free parameters. To conclude, these analyses considered a small sample of dispersion-dominated galaxies, as opposed to our larger sample of rotation-dominated galaxies. Their best-fit ULDM mass $m \lesssim 10^{-22} \mathrm{eV}$ is disfavored by our findings.

Reference [26] fitted ULDM soliton + halo profiles to SPARC [28] galaxies. Without a soliton-host halo relation, Ref. [26] assigned separate free parameters to the NFW halo and to the soliton, for each galaxy. As a result of this freedom, it is difficult to understand from that analysis if ULDM is ruled out or not in the mass range $m=\left(10^{-22} \div 10^{-21}\right) \mathrm{eV}$. Nevertheless, Ref. [26] did state a best fit of $m \approx 0.5 \times 10^{-23} \mathrm{eV}$, and noted the tension with Ly- $\alpha$ data, in agreement with our findings. Our work here shows that the soliton-host halo relation reduces the modeling freedom, and leads to disagreement with rotation curve data.

Recently, Ref. [59] addressed the problem from a different angle. It builds on the results of Ref. [60] that fitted rotations curves of a sample of galaxies assuming that their halos are described by the Burkert profile. Reference [59] points out a correlation between the parameters of the fitted profiles-the central dark matter density $\rho_{c}$ and the size of the core $R_{c}$,

$$
\rho_{c} \propto R_{c}^{-\beta}
$$

with $\beta \sim 1$. This is different from $\beta=4$ predicted by ULDM if the cores are identified with the solitons, see Eq. (30). Thus, Ref. [59] concludes that ULDM cannot explain the origin of the halo cores, and cannot solve the core-cusp problem of $\Lambda \mathrm{CDM}$. Our results are consistent with these findings and strengthen them by showing that the soliton-host halo relation implies a tension between predictions of ULDM and the data for $m \sim\left(10^{-22} \div 10^{-21}\right) \mathrm{eV}$, disfavoring this mass range altogether.

\section{B. Caveats}

\section{Soliton formation time}

Our analysis has been based on the assumption that ULDM solitons satisfying the soliton-host halo relation exist in the centers of all (or most) galactic halos. Formation of such solitons within the lifetime of the universe has been demonstrated in numerical simulations $[6,61]$ for ULDM masses $m \sim 10^{-22} \mathrm{eV}$ and halos with mass $\left(10^{9} \div 10^{11}\right) M_{\odot}$. Here we discuss whether such solitons have enough time to form for higher values of $m$ and larger halos.

Recently, Ref. [62] considered the scenario, where solitons form as a result of gravitational relaxation of the inner part of the halo. In this case, the relaxation time can be estimated as

$$
\tau_{\text {relax }}=\frac{b \sqrt{2} m^{3} v^{6}}{12 \pi^{3} G^{2} \rho^{2} \Lambda},
$$

where $v$ and $\rho$ are the mean velocity and density in the inner part of the halo, $\Lambda=\log (m v R)$ is the Coulomb logarithm depending on the size $R$ of the relaxation region, and $b$ is a numerical coefficient close to one. The formula has been confirmed in [62] by numerical simulations that found $b$ to range between $0.7 \div 0.9$ depending on the precise form of the initial conditions. Substituting into Eq. (61) the values for a typical halo and taking $\Lambda \sim 4$, we obtain,

$$
\begin{aligned}
\tau_{\text {relax }} \sim & 7 \times 10^{8}\left(\frac{m}{10^{-22} \mathrm{eV}}\right)^{3} \\
\times & \times\left(\frac{v}{100 \mathrm{~km} / \mathrm{s}}\right)^{6}\left(\frac{\rho}{0.1 M_{\odot} / \mathrm{pc}^{3}}\right)^{-2} \mathrm{yr} .
\end{aligned}
$$

We observe that the relaxation time has strong dependence on the velocity and the density in the central region of the halo, that have large uncertainties. Fixing the fiducial values of $v=100 \mathrm{~km} / \mathrm{s}$ and $\rho=0.1 M_{\odot} / \mathrm{pc}^{3}$ one would conclude that the relaxation takes longer than the age of the universe if $m \gtrsim 3 \times 10^{-22} \mathrm{eV}$.

However, it can be incorrect to use Eq. (61) for the estimate of the soliton formation time inside ULDM halos in the cosmological context. Indeed, this equation has been derived and tested in the kinetic regime corresponding to large velocity dispersion of ULDM particles, such that their de Broglie wavelength is much shorter than the size of the system. Starting from initial conditions of a gas of wavepackets with large velocity dispersion in a box, Ref. [62] observed a fast formation of a virialized halo, followed by a long period of kinetic evolution populating the low-lying energy levels, until the system was able to form the soliton through Bose-Einstein condensation. The time scale (61) refers to the long second stage of the process. On the other hand, the cosmological initial conditions prior to virialization are characterized by very low velocities, where the kinetic description does not apply. In this situation, a long relaxation seems unnecessary and the formation of the soliton can proceed much faster, on the scale of the halo free-fall time; see discussion in [62]. This is supported by the simulations with cosmological initial conditions which appear to imply formation of the soliton in every halo at the moment of virialization $[6,61]$. We believe that the issue of the soliton formation time requires further investigation, including possible effects of baryonic matter.

\section{Nongravitational interactions}

In our analysis, we have neglected any nongravitational interactions of the ULDM particles. Let us discuss under which conditions this approximation is justified. As an example we consider quartic self-interaction of the scalar field $\phi$ with coupling constant $\kappa$, 


$$
\delta V(\phi)=\frac{\kappa \phi^{4}}{4}
$$

The self-interaction is negligible as long as the corresponding potential energy is much smaller than the gradient energy of the field,

$$
\frac{|\kappa| \phi^{4}}{4} \ll \frac{(\nabla \phi)^{2}}{2} .
$$

For a soliton with the scale parameter $\lambda$, this translates into a bound,

$$
|\kappa|<\frac{2 m^{2}}{x_{c \lambda}^{2} \rho_{c \lambda}} .
$$

Using the soliton-host halo relation in the form of Eqs. (29), (30) we obtain the condition,

$$
|\kappa|<4 \times 10^{-93}\left(\frac{m}{10^{-22} \mathrm{eV}}\right)^{2}\left(\frac{M_{h}}{10^{12} M_{\odot}}\right)^{-\frac{2}{3}} .
$$

If this condition is satisfied, our analysis is applicable. Otherwise, the effects of the self-interaction on the soliton properties must be taken into account.

While the condition (66) appears stringent, it is naturally fulfilled in a broad class of theories containing axionlike particles. These typically have a periodic potential of the form,

$$
V(\phi)=m^{2} f^{2}(1-\cos (\phi / f)) .
$$

For the masses of interest $m \sim\left(10^{-22} \div 10^{-18}\right) \mathrm{eV}$, the axion periodicity should be in the range $f \sim\left(10^{16} \div\right.$ $\left.10^{17}\right) \mathrm{GeV}$ to produce the right dark matter abundance via the misalignment mechanism (see e.g., [12]). Expanding the potential (67) in powers of $\phi$ we obtain the value of the coupling,

$$
\begin{aligned}
\kappa & =-\frac{m^{2}}{6 f^{2}} \\
& \approx-1.7 \times 10^{-97}\left(\frac{m}{10^{-22} \mathrm{eV}}\right)^{2}\left(\frac{f}{10^{17} \mathrm{GeV}}\right)^{-2} .
\end{aligned}
$$

We see that (66) is indeed satisfied for all galactic halos considered in this paper, independently of the ULDM mass.

Similar analysis can be performed in the case of nongravitational interactions between ULDM and baryonic matter, though in this case it will be strongly modeldependent.

\section{SUMMARY}

Bosonic ultralight dark matter (ULDM) is an interesting paradigm for dark matter. For particle mass $m \sim 10^{-22} \mathrm{eV}$,
ULDM would form solitonic cores in the center of galaxies, and it has been suggested that this could solve several puzzles of $\Lambda$ CDM on small scales.

We analyzed the results of numerical simulations of ULDM, which have found scaling relations between the mass of the central soliton and the mass or energy of the host halo. Simulations by different groups converge on a soliton profile in good agreement with the analytical solution of the Schroedinger-Poisson (SP) equation, admitting important analytic insight into the numerical results.

We showed that the simulations of Ref. [13] contain a central soliton that dominates the total energy of the entire soliton + halo system. This situation is unlikely to describe realistic galactic halos ${ }^{9}$ with mass above $\sim 5 \times 10^{7}\left(\mathrm{~m} / 10^{-22} \mathrm{eV}\right)^{-3 / 2} \mathrm{M}_{\odot}$.

We have demonstrated that the soliton-host halo relation found in the simulations of Refs. [6,7] can be summarized by the statement, that $\left.(E / M)\right|_{\text {soliton }}=\left.(E / M)\right|_{\text {halo }}$. The simulations of [7] show a small spread, less than a factor of two, around this relation. This $E / M$ relation could apply to real galaxies.

The $E / M$ soliton-host halo relation implies that the peak circular velocity of the large-scale halo should approximately reproduce itself in the inner soliton region. This can be tested against observations without free parameters. Contrasting this prediction with high-resolution disc galaxy data, we showed that ULDM in the mass range $m \sim$ $\left(10^{-22} \div 10^{-21}\right) \mathrm{eV}$ is disfavored by observations. Given that smaller $m$ is in tension with cosmological measurements, this disfavors ULDM as a solution to the small scale puzzles of $\Lambda \mathrm{CDM}$.

We also analyzed the effect of a fixed background distribution of baryons and of a super-massive black hole (SMBH) on the soliton solution. We showed that this analysis would be important towards using high-resolution kinematical data in the Milky Way or Milky Way-like galaxies. Mapping the Milky Way gravitational potential down to $r \sim 10^{-2}$ pc may allow us to probe ULDM with mass up to $m \sim 10^{-19} \mathrm{eV}$.

It should be stressed that the $E / M$ soliton-host halo relation (35) is an empirical result, deduced from numerical simulations without baryons and tested in a limited range of ULDM and halo masses. The importance of its phenomenological implications motivates further investigation to better understand the physics underlying this relation and map out its domain of validity.

Throughout the paper we have assumed that ULDM comprises the total amount of dark matter. We leave for future the study of scenarios where only a fraction of dark matter is in the form of ULDM.

\footnotetext{
${ }^{9}$ This does not invalidate the beautiful simulations of [13]. We only think that their setup for initial conditions does not match real galaxies.
} 


\section{ACKNOWLEDGMENTS}

We thank James Hung-Hsu Chan, Josh Eby, Lam Hui, Dmitry Levkov, Moti Milgrom, Eran Ofek, Alexander Panin, and Igor Tkachev for discussions, and Yossi Nir, Scott Tremaine, and Eli Waxman for comments on an early version of the draft. K. B. thanks the participants of the VBSM Polynesia workshop, where part of this work was conducted, for useful discussions. K. B. is incumbent of the Dewey David Stone and Harry Levine career development chair. The work of K. B. and N. B. is supported by Grant No. 1937/12 from the I-CORE program of the Planning and Budgeting Committee and the Israel Science Foundation and by Grant No. 1507/16 from the Israel Science Foundation. S. S. is grateful to Columbia University for hospitality during completion of this work.

\section{APPENDIX A: SOLITON MASS-HOST HALO MASS RELATION, $M_{h}$ vs $M_{200}$}

Reference [7] defined the halo virial mass $M_{h}$ and radius $R_{v}$,

$$
M_{h}=\frac{4 \pi}{3} R_{v}^{3} \zeta(z) \rho_{m 0},
$$

where $\rho_{m 0}$ is the present matter density and $\zeta(z)=$ $\left(18 \pi^{2}-82\left(1-\Omega_{m}(z)\right)-39\left(1-\Omega_{m}(z)\right)^{2}\right) / \Omega_{m}(z)$. The halo energy per unit mass was estimated as $E_{h} / M_{h}=$ $-3 G M_{h} /\left(10 R_{v}\right)=-G \frac{3}{10}\left(\frac{4 \pi}{3} \zeta \Omega_{m 0}\right)^{\frac{1}{3}} \rho_{c}^{\frac{1}{3}} M_{h}^{\frac{2}{3}}$. In Sec. IV, we prefer to work with $M_{200}$ and $R_{200}$ and have calculated the halo energy per unit mass, using Eq. (42), as $E_{200} / M_{200}=-G \frac{\tilde{c}}{4}\left(\frac{4 \pi \delta_{c}}{\left(\ln (1+c)-\frac{c}{1+c}\right)^{2}}\right)^{\frac{1}{3}} \rho_{c}^{\frac{1}{3}} M_{200}^{\frac{2}{3}}$.

To express the soliton mass-host halo mass relation, Eq. (45), in terms of $M_{h}$ of Ref. [7], we need to do so at fixed $E_{200} / M_{200}=E_{h} / M_{h} \equiv E / M$. This gives:

$$
\left.\left(\frac{M_{200}}{M_{h}}\right)^{\frac{1}{3}}\right|_{E / M}=\left(\frac{\frac{3}{10}\left(\frac{4 \pi}{3} \zeta \Omega_{m 0}\right)^{\frac{1}{3}}}{\frac{\tilde{c}}{4}\left(\frac{4 \pi \delta_{c}}{\left(\ln (1+c)-\frac{c}{1+c}\right)^{2}}\right)^{\frac{1}{3}}}\right)^{\frac{1}{2}} .
$$

For $c$ in the range $5 \div 30$, the RHS of Eq. (A2) varies between 0.58 and 0.47 . Plugging this result into Eq. (45) shows that it agrees with Eq. (31) of [7] to $20 \%$.

\section{APPENDIX B: Adding a super-massive black hole}

Most galaxies, if not all, host a SMBH, and we should check how it affects the ULDM soliton. This is an important point to verify and we do this analysis here in some detail. The upshot of our discussion is that a SMBH would not affect our results for small disc galaxies, whereas in the MW it becomes relevant at the upper end of considered ULDM masses, $m \gtrsim 5 \times 10^{-20} \mathrm{eV}$.

We start with a Newtonian analysis, dealing with the ULDM configuration far from the $\mathrm{BH}$ Schwarzschild radius. Then we will consider the limitations of this analysis imposed by absorption of ULDM on SMBH that leads to the decay of the soliton $+\mathrm{SMBH}$ configuration.

\section{Soliton shape in the presence of SMBH}

Adding a SMBH, coincident with the soliton center of mass, changes Eqs. (7)-(8) into

$$
\begin{gathered}
\partial_{r}^{2}(r \chi)=2 r\left(\Phi-\frac{A}{r}-\gamma\right) \chi \\
\partial_{r}^{2}(r \Phi)=r \chi^{2},
\end{gathered}
$$

where

$$
\begin{aligned}
A & =G M_{\mathrm{BH}} m \\
& \approx 3 \times 10^{-6}\left(\frac{M_{\mathrm{BH}}}{4 \times 10^{6} \mathrm{M}_{\odot}}\right)\left(\frac{m}{10^{-22} \mathrm{eV}}\right) .
\end{aligned}
$$

We chose the reference value for $M_{\mathrm{BH}}$ to represent the case of the MW.

It remains convenient to solve the problem using boundary conditions with $\chi(0)=1$. Let us denote this solution (satisfying $\chi(0)=1$ ) by $\chi_{1}(r ; A)$, accompanied by the potential $\Phi_{1}(r ; A)$. Having found $\chi_{1}(r ; A)$ and $\Phi_{1}(r ; A)$ for any value of $A$, the physical solution $\chi_{\lambda}(r ; A)$ satisfying Eqs. (B1)-(B2) with boundary condition $\chi_{\lambda}(0 ; A)=\lambda^{2}$ is given by

$$
\begin{gathered}
\chi_{\lambda}(r ; A)=\lambda^{2} \chi_{1}(\lambda r ; A / \lambda), \\
\Phi_{\lambda}(r ; A)=\lambda^{2} \Phi_{1}(\lambda r ; A / \lambda) .
\end{gathered}
$$

Defining $M_{\lambda}(A)$, with obvious notation, the soliton mass is

$$
M_{\lambda}(A)=\lambda M_{1}(A / \lambda) .
$$

Figure 17 shows the density profile $\chi_{1}^{2}(r ; A)$ for different values of $A$. For $A \lesssim 10^{-2}$ the solution converges to the unperturbed $\chi_{1}$. For $A \gtrsim 1$ the solution approaches $\chi_{1}(r ; A \rightarrow \infty) \rightarrow e^{-A r}$ which is nothing, but the wavefunction of the ground state in the Coulomb potential. This means that for $A / \lambda \gtrsim 1$, solitons with different values of $\lambda$ have the same profile up to over-all normalization: $\chi_{\lambda}(r ; A \gtrsim \lambda) \rightarrow \lambda^{2} e^{-A r}$. In this limit, the soliton core radius is $r_{c \lambda}(A) \rightarrow \ln (2) /(2 A)$, and the mass is $M_{\lambda}(A) \rightarrow \frac{\lambda^{4}}{4 A^{3}} \frac{M_{\mathrm{pl}}^{2}}{m}$. More generally, for any $A$ the mass-radius relation is

$$
M_{\lambda}(A) r_{c \lambda}(A)=M_{1}(A / \lambda) r_{c 1}(A / \lambda) .
$$

The mass-radius product is shown by the solid blue curve in Fig. 18 (top panel). The large- $A$ analytic solution, $\left.M_{1}(A) r_{c 1}(A)\right|_{A \rightarrow \infty} \rightarrow \frac{\ln (2)}{8 A^{4}} \frac{M_{\mathrm{pl}}^{2}}{m}$ (recall $r=m x$ ), is shown in dotted black, normalized to the $A=0$ result.

As discussed in Sec. III A, Eqs. (31) and (35) are equivalent when the ULDM soliton is self-gravitating, but this equivalence is broken by the SMBH gravitational potential. It is unlikely that Eq. (31) remains realistic when 


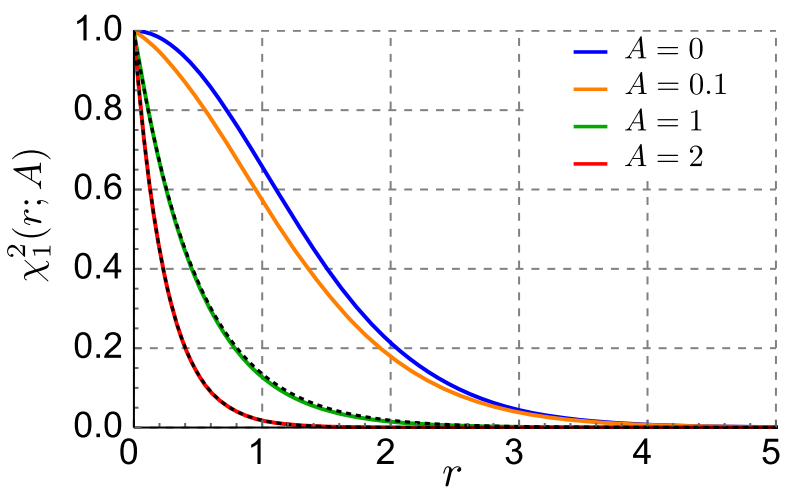

FIG. 17. $\chi_{1}^{2}(A)$ profiles, with $A=0,0.1,1,2$ shown in blue, orange, green and red, respectively. For the $A=1$ and $A=2$ profiles, the large- $A$ analytic solution is shown in dotted black.

the SMBH becomes important. Instead, Eq. (35) may still be valid. In computing the ULDM energy, we need to account for the SMBH contribution,

$$
E(A)=\int d^{3} x\left(\frac{|\nabla \psi|^{2}}{2 m^{2}}+\left(\frac{\Phi}{2}-\frac{A}{m x}\right)|\psi|^{2}\right),
$$

which means that

$$
E_{\lambda}(A)=\lambda^{3} E_{1}(A / \lambda)
$$

The large $A$ limit (attained for a $\chi_{\lambda}(r ; A)$ soliton at $A \gtrsim \lambda$ ) is $E_{\lambda}(A \rightarrow \infty) \rightarrow-\frac{\lambda^{4}}{8 A} \frac{M_{\mathrm{pl}}^{2}}{m}$. In this limit, $E_{\lambda} / M_{\lambda}$ tends to a $\lambda$ independent constant, $E_{\lambda} / M_{\lambda}(A \rightarrow \infty) \rightarrow-A^{2} / 2$, because the soliton is not held together by self gravity, but by the SMBH potential. The $E / M$ relation for the $\chi_{1}(A)$ soliton is shown in the bottom panel of Fig. 18.

In Fig. 19, solid lines show $E / M$ for the soliton with different values of particle mass $m$, in the presence of a SMBH with $M_{\mathrm{BH}}=4 \times 10^{6} \mathrm{M}_{\odot}$. The dashed lines show the undistorted result, obtained from Eq. (25). The large $A$ limit with constant $E_{\lambda} / M_{\lambda}$ is attained when the soliton mass becomes smaller than the SMBH mass.

To further clarify this point we relate $A / \lambda$ to the unperturbed soliton mass using Eq. (15),

$$
\begin{aligned}
A / \lambda & \approx 2.06\left(\frac{M_{\mathrm{BH}}}{M_{\lambda}(A=0)}\right) \\
& \approx 0.8\left(\frac{M_{\mathrm{BH}}}{4 \times 10^{6} \mathrm{M}_{\odot}}\right)\left(\frac{M_{\lambda}(A=0)}{10^{7} \mathrm{M}_{\odot}}\right)^{-1} .
\end{aligned}
$$

Next, we see from Eqs. (B4), (B5) that $A / \lambda$ gives an effective $A$-parameter that must be substituted in the $\chi_{1^{-}}$ soliton to obtain the actual solution. Thus, whether the effects of SMBH are important or not can be read from the ratio of the SMBH mass to the mass of the undistorted soliton.

To analyze the rotation curve for the soliton $+\mathrm{SMBH}$ system, it is useful to view $V_{\text {circ }}$ for the $\chi_{1}(A)$ soliton, obtained for different values of $A$. This is shown in Fig. 20.
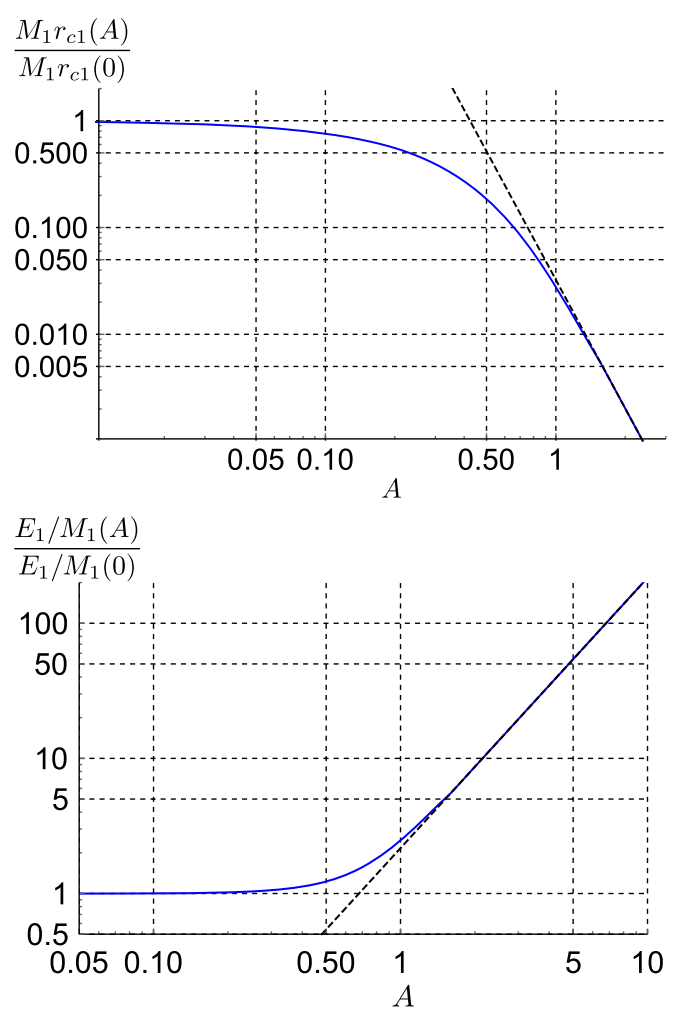

FIG. 18. Mass-core radius relation (top panel) and $E / M$ (bottom panel) for the $\chi_{1}(A)$ soliton, as function of $A$. The large- $A$ analytic solution is shown in dashed black.

Because $\chi_{\lambda}(A)$ solitons are a scale transformation of $\chi_{1}(A / \lambda)$, plotting $\chi_{1}(A)$ with different values of $A$ is equivalent to plotting the rescaled velocity curve of solitons at different values of $M_{\mathrm{BH}} / M_{\lambda}$. For example, using Eq. (B10), the $A=0.2$ curve in Fig. 20 corresponds to a soliton with $M_{\lambda} \approx 10 M_{\mathrm{BH}}$; while the $A=10^{-2}$ corresponds to $M_{\lambda} \approx 200 M_{\mathrm{BH}}$.

For $M_{\lambda} \gtrsim 10 M_{\mathrm{BH}}$, the soliton's circular velocity peak and the peak location are affected very little by the SMBH.

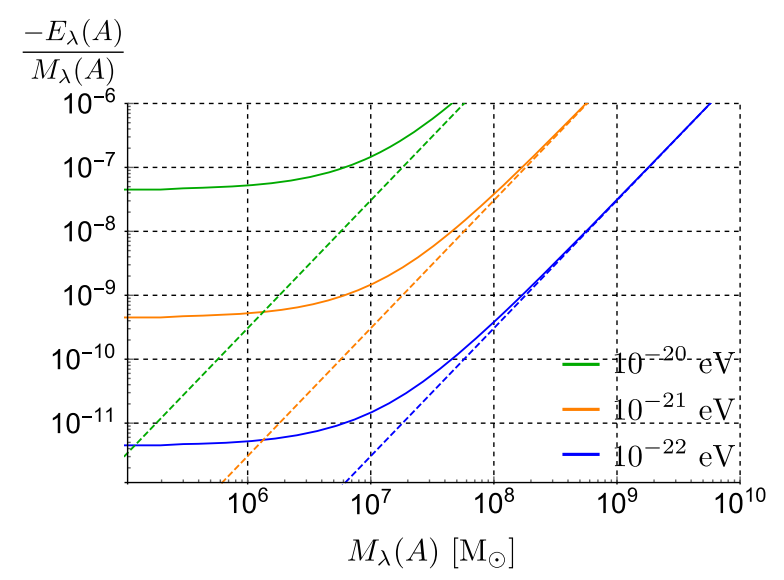

FIG. 19. Solid lines: energy per unit mass vs mass for a soliton, including the effect of a SMBH with $M_{\mathrm{BH}}=4 \times 10^{6} \mathrm{M}_{\odot}$. Dashed lines: switching off the SMBH. 


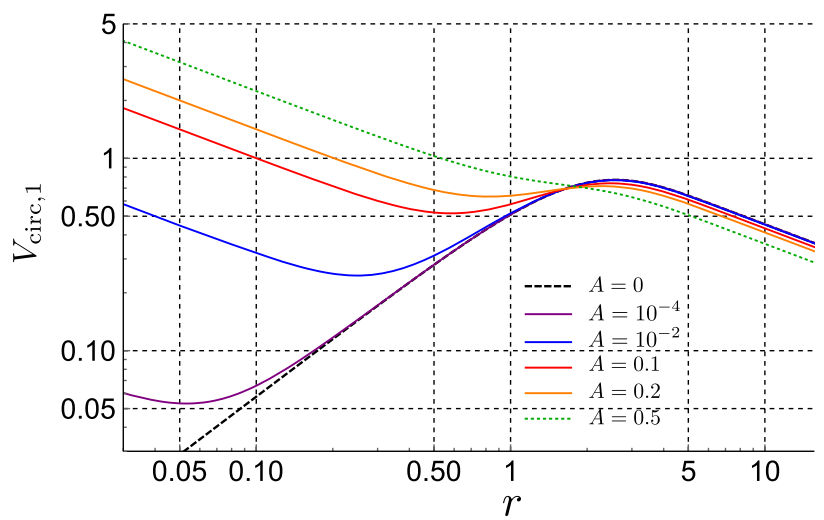

FIG. 20. Rotation curve for the $\chi_{1}(A)$ soliton.

However, at small $x$ approaching the SMBH, the velocity curve turns back up reflecting the gravitational potential of the SMBH itself. This implies that the rotation curve of a galaxy hosting a SMBH may not decrease appreciably below $x_{\text {peak }, \lambda}$, even when $M_{\mathrm{BH}}$ is much smaller than $M_{\lambda}$. For example, even for $M_{\mathrm{BH}}=0.05 M_{\lambda}(A=0.1$ in Fig. 20), the circular velocity decreases by only $\sim 25 \%$ at $x<x_{\text {peak }, \lambda}$ before it goes back up due to the BH. For the same parameters, Fig. 18 shows that $E_{\lambda} / M_{\lambda}$ is essentially unperturbed, meaning that the soliton-host halo relation of Eq. (35) and the rotation curve properties discussed in Sec. IV remain unaffected. Such a galaxy would have an approximately flat rotation curve all the way in to the region of SMBH dominance.

The impact of a SMBH on the analysis of Sec. IV can be concluded as follows. For $M_{\lambda} \gtrsim 4 M_{\mathrm{BH}}$ (corresponding, by using Eq. (B10), to effective $A / \lambda \approx 0.5$ ), Fig. 18 shows that $E_{\lambda} / M_{\lambda}$ is corrected by $\lesssim 25 \%$ compared to its unperturbed value. Equations (28) and (44) then imply that $\lambda$ and $\max V_{\text {circ }, \lambda}$ are corrected by an insignificant $\sim 12 \%$. For a large SMBH with $M_{\mathrm{BH}} \gtrsim M_{\lambda}$, on the other hand, the SMBH gravitational potential itself must produce high rotation velocity at $x_{\text {peak }, \lambda}$, comparable or higher than what the soliton itself would provide. As a result, besides from the fact that the rotation curve may not decrease towards lower $x$ below the soliton peak, our analysis in and observational limits from Sec. IV should be robust against the effect of a SMBH. ${ }^{10}$

\section{Absorption of soliton by SMBH}

We now discuss accretion of ULDM from the soliton onto SMBH. To estimate the lifetime of the soliton + SMBH configuration we follow the approach of [12] (see also $[64,65]$ for the study of scalar bound states in external

\footnotetext{
${ }^{10}$ This conclusion seems in contradiction with the claim of Ref. [63], who proposed that ULDM solitons distorted by SMBHs could explain the $M_{\mathrm{BH}}-\sigma$ correlation, observed between SMBHs and the stellar bulge of the galaxies hosting them.
}

Schwarzschild metric and [66] for a numerical analysis of the lifetime of soliton $+\mathrm{BH}$ system). Unruh [67] has derived the cross section for absorption of a scalar particle with mass $m$ and momentum $k$ by a Schwarzschild $\mathrm{BH}$, whose size is much smaller than the Compton wavelength of the particle. In the nonrelativistic limit, $k \ll m$, it has the form,

$$
\sigma=\frac{32 \pi^{2}\left(G M_{\mathrm{BH}}\right)^{3} m^{3}}{k^{2}\left(1-e^{-\zeta}\right)}
$$

where

$$
\zeta=2 \pi G M_{\mathrm{BH}} m^{2} / k
$$

As the cross section is s-wave dominated, it is appropriate for calculation of accretion from a spherically symmetric scalar field configuration.

The growth of $\mathrm{BH}$ mass due to an infalling flux of particles with density $\rho$ is,

$$
\frac{d M_{\mathrm{BH}}}{d t}=\frac{32 \pi^{2}\left(G M_{\mathrm{BH}}\right)^{3} m^{2} \rho}{k\left(1-e^{-\zeta}\right)} .
$$

In the case of the soliton, we can estimate the absorption rate by substituting into this expression the central density of the soliton and the characteristic momentum of particles in the soliton that can be read from the soliton profile (30),

$$
k \sim 0.3 x_{c}^{-1} .
$$

This approximation is justified as long as the mass of the soliton is bigger than the $\mathrm{BH}$ mass, so that the effect of $\mathrm{BH}$ on the soliton shape can be neglected.

The expression for the accretion rate simplifies in the limiting cases when the parameter $\zeta$ is small or large. Using Eq. (29) (which is consistent with the soliton-host halo relation) we obtain,

$$
\zeta=0.16\left(\frac{m}{10^{-22} \mathrm{eV}}\right)\left(\frac{M_{\mathrm{BH}}}{4 \times 10^{6} M_{\odot}}\right)\left(\frac{M_{h}}{10^{12} M_{\odot}}\right)^{-\frac{1}{3}} .
$$

If $\zeta \ll 1$, the characteristic accretion time, during which the $\mathrm{BH}$ mass grows by a factor 2 is,

$$
\begin{aligned}
\tau_{\zeta \ll 1} \sim & 2.4 \times 10^{17}\left(\frac{m}{10^{-22} \mathrm{eV}}\right)^{-2} \\
& \times\left(\frac{M_{\mathrm{BH}}}{4 \times 10^{6} M_{\odot}}\right)^{-1}\left(\frac{M_{h}}{10^{12} M_{\odot}}\right)^{-\frac{4}{3}} \mathrm{yr} .
\end{aligned}
$$

In the opposite regime, $\zeta \gg 1$, the characteristic time is 


$$
\begin{aligned}
\tau_{\zeta \gg 1} \sim & 1.5 \times 10^{18}\left(\frac{m}{10^{-22} \mathrm{eV}}\right)^{-3} \\
& \times\left(\frac{M_{\mathrm{BH}}}{4 \times 10^{6} M_{\odot}}\right)^{-2}\left(\frac{M_{h}}{10^{12} M_{\odot}}\right)^{-1} \mathrm{yr} .
\end{aligned}
$$

In deriving these expressions, we made use of Eqs. (29) and (30). Both expressions give the accretion time longer than the age of the universe for MW's SMBH with $M_{\mathrm{BH}} \approx$ $4.3 \times 10^{6} \mathrm{M}_{\odot}$ and $m \lesssim 5 \times 10^{-20} \mathrm{eV}$. Our Newtonian analysis, addressing stationary ULDM soliton solutions, is adequate in this case. For galaxies with a more massive SMBH, e.g., M31 with $M_{\mathrm{BH}} \sim 10^{8} \mathrm{M}_{\odot}$ [68], Eq. (B16) implies that absorption of the ULDM field into the SMBH becomes important already for $m \gtrsim 6 \times 10^{-21} \mathrm{eV}$, and our stationary Newtonian analysis is not expected to capture the physics correctly for such values of $m$. Finally, for the reference value of $m=10^{-22} \mathrm{eV}$, our analysis should be valid as long as $M_{\mathrm{BH}} \lesssim 10^{10} \mathrm{M}_{\odot}$.
[1] W. Hu, R. Barkana, and A. Gruzinov, Cold and Fuzzy Dark Matter, Phys. Rev. Lett. 85, 1158 (2000).

[2] A. Arbey, J. Lesgourgues, and P. Salati, Quintessential haloes around galaxies, Phys. Rev. D 64, 123528 (2001).

[3] J. Lesgourgues, A. Arbey, and P. Salati, A light scalar field at the origin of galaxy rotation curves, New Astron. Rev. 46, 791 (2002).

[4] P.-H. Chavanis, Mass-radius relation of Newtonian selfgravitating Bose-Einstein condensates with short-range interactions: I. Analytical results, Phys. Rev. D 84, 043531 (2011).

[5] P. H. Chavanis and L. Delfini, Mass-radius relation of Newtonian self-gravitating Bose-Einstein condensates with short-range interactions: II. Numerical results, Phys. Rev. D 84, 043532 (2011).

[6] H.-Y. Schive, T. Chiueh, and T. Broadhurst, Cosmic structure as the quantum interference of a coherent dark wave, Nat. Phys. 10, 496 (2014).

[7] H.-Y. Schive, M.-H. Liao, T.-P. Woo, S.-K. Wong, T. Chiueh, T. Broadhurst, and W. Y. P. Hwang, Understanding the Core-Halo Relation of Quantum Wave Dark Matter from 3D Simulations, Phys. Rev. Lett. 113, 261302 (2014).

[8] D. J. E. Marsh and A.-R. Pop, Axion dark matter, solitons and the cusp-core problem, Mon. Not. R. Astron. Soc. 451, 2479 (2015).

[9] S.-R. Chen, H.-Y. Schive, and T. Chiueh, Jeans analysis for Dwarf Spheroidal Galaxies in wave dark matter, Mon. Not. R. Astron. Soc. 468, 1338 (2017).

[10] B. Schwabe, J. C. Niemeyer, and J. F. Engels, Simulations of solitonic core mergers in ultralight axion dark matter cosmologies, Phys. Rev. D 94, 043513 (2016).

[11] J. Veltmaat and J. C. Niemeyer, Cosmological particle-incell simulations with ultralight axion dark matter, Phys. Rev. D 94, 123523 (2016).

[12] L. Hui, J. P. Ostriker, S. Tremaine, and E. Witten, Ultralight scalars as cosmological dark matter, Phys. Rev. D 95, 043541 (2017).

[13] P. Mocz, M. Vogelsberger, V.H. Robles, J. Zavala, M. Boylan-Kolchin, A. Fialkov, and L. Hernquist, Galaxy formation with BECDM: I. Turbulence and relaxation of idealized haloes, Mon. Not. R. Astron. Soc. 471, 4559 (2017).
[14] P. Svrcek and E. Witten, Axions in string theory, J. High Energy Phys. 06 (2006) 051.

[15] A. Arvanitaki, S. Dimopoulos, S. Dubovsky, N. Kaloper, and J. March-Russell, String axiverse, Phys. Rev. D 81, 123530 (2010).

[16] D. J. E. Marsh, Axion cosmology, Phys. Rep. 643, 1 (2016).

[17] A. Del Popolo and M. Le Delliou, Small scale problems of the $\Lambda$ CDM model: A short review, Galaxies 5, 17 (2017).

[18] B. Bozek, D. J. E. Marsh, J. Silk, and R. F. G. Wyse, Galaxy UV-luminosity function and reionization constraints on axion dark matter, Mon. Not. R. Astron. Soc. 450, 209 (2015).

[19] E. Armengaud, N. Palanque-Delabrouille, D. J. E. Marsh, J. Baur, and C. Yche, Constraining the mass of light bosonic dark matter using SDSS Lyman- $\alpha$ forest, Mon. Not. R. Astron. Soc. 471, 4606 (2017).

[20] V. Iršič, M. Viel, M. G. Haehnelt, J. S. Bolton, and G. D. Becker, First Constraints on Fuzzy Dark Matter from Lyman- $\alpha$ Forest Data and Hydrodynamical Simulations, Phys. Rev. Lett. 119, 031302 (2017).

[21] J. Zhang, J.-L. Kuo, H. Liu, Y.-L. S. Tsai, K. Cheung, and M.-C. Chu, Is fuzzy dark matter in tension with Lymanalpha forest?, Astrophys. J. 863, 73 (2018).

[22] T. Kobayashi, R. Murgia, A. De Simone, V. Iršič, and M. Viel, Lyman-alpha constraints on ultralight scalar dark matter: Implications for the early and late universe, Phys. Rev. D 96, 123514 (2017).

[23] E. Calabrese and D. N. Spergel, Ultra-light dark matter in ultra-faint dwarf galaxies, Mon. Not. R. Astron. Soc. 460, 4397 (2016).

[24] A. X. Gonzáles-Morales, D. J. E. Marsh, J. Peñarrubia, and L. Ureña López, Unbiased constraints on ultralight axion mass from dwarf spheroidal galaxies, Mon. Not. R. Astron. Soc. 472, 1346 (2017).

[25] V. H. Robles and T. Matos, Flat central density profile and constant DM surface density in galaxies from scalar field dark matter, Mon. Not. R. Astron. Soc. 422, 282 (2012).

[26] T. Bernal, L. M. Fernández-Hernández, T. Matos, and M. A. Rodrguez-Meza, Rotation curves of high-resolution LSB and SPARC galaxies in wave (fuzzy) and multistate (ultralight boson) scalar field dark matter, Mon. Not. R. Astron. Soc. 475, 1447 (2018). 
[27] W. J. G. de Blok and A. Bosma, High-resolution rotation curves of low surface brightness galaxies, Astron. Astrophys. 385, 816 (2002).

[28] F. Lelli, S. S. McGaugh, and J. M. Schombert, SPARC: Mass models for 175 disk galaxies with spitzer photometry and accurate rotation curves, Astron. J. 152, 157 (2016).

[29] J. H. H. Chan, H.-Y. Schive, T.-P. Woo, and T. Chiueh, How do stars affect $\psi \mathrm{DM}$ halos?, Mon. Not. R. Astron. Soc. 478, 2686 (2018).

[30] T. Rindler-Daller, T. Rindler-Daller, P. R. Shapiro, and P. R. Shapiro, Finding new signature effects on galactic dynamics to constrain bose-einstein-condensed cold dark matter, Astrophys. Space Sci. Proc. 38, 163 (2014).

[31] V. Desjacques, A. Kehagias, and A. Riotto, Impact of ultralight axion self-interactions on the large scale structure of the Universe, Phys. Rev. D 97, 023529 (2018).

[32] R. Ruffini and S. Bonazzola, Systems of selfgravitating particles in general relativity and the concept of an equation of state, Phys. Rev. 187, 1767 (1969).

[33] T.-P. Woo and T. Chiueh, High-resolution simulation on structure formation with extremely light bosonic dark matter, Astrophys. J. 697, 850 (2009).

[34] J. A. Eby, Phenomenology and astrophysics of gravitationally-bound condensates of axion-like particles, Ph. D. thesis, Cincinnati University, 2017, http://lss.fnal.gov/archive/ thesis/2000/fermilab-thesis-2017-19.pdf.

[35] M. Boylan-Kolchin, V. Springel, S. D. M. White, and A. Jenkins, There's no place like home? Statistics of Milky Way-mass dark matter halos, Mon. Not. R. Astron. Soc. 406, 896 (2010).

[36] T. Piffl et al., Constraining the Galaxy's dark halo with RAVE stars, Mon. Not. R. Astron. Soc. 445, 3133 (2014).

[37] J. Vicens, J. Salvado, and J. Miralda-Escudé, Bosonic dark matter halos: Excited states and relaxation in the potential of the ground state, arXiv:1802.10513.

[38] A. M. Ghez, E. Becklin, G. Duchene, S. D. Hornstein, M. Morris, S. Salim, and A. Tanner, Full three dimensional orbits for multiple stars on close approaches to the central supermassive black hole, Astron. Nachr. 324, 527 (2003).

[39] A. Feldmeier-Krause, L. Zhu, N. Neumayer, G. van de Ven, P. T. de Zeeuw, and R. Schdel, Triaxial orbit-based modelling of the milky way nuclear star cluster, Mon. Not. R. Astron. Soc. 466, 4040 (2017).

[40] M. T. McGinn, K. Sellgren, E. E. Becklin, and D. N. B. Hall, Stellar kinematics in the galactic center, Astrophys. J. 338, 824 (1989).

[41] M. Lindqvist, H. J. Habing, and A. Winnberg, Oh/ir stars close to the galactic centre. ii-their spatial and kinematic properties and the mass distribution within 5-100 pc from the galactic centre, Astron. Astrophys. 259, 118 (1992).

[42] S. Deguchi, H. Imai, T. Fujii, I. S. Glass, Y. Ita, H. Izumiura, O. Kameya, A. Miyazaki, Y. Nakada, and J.-I. Nakashima, Sio maser survey of the large-amplitude variables in the galactic center, Publ. Astron. Soc. Jpn. 56, 261 (2004).

[43] S. Trippe, S. Gillessen, O. E. Gerhard, H. Bartko, T. K. Fritz, H. L. Maness, F. Eisenhauer, F. Martins, T. Ott, K. DoddsEden, and R. Genzel, Kinematics of the old stellar population at the galactic centre, Astron. Astrophys. 492, 419 (2008).
[44] S. Oh, S. S. Kim, and D. F. Figer, Mass distribution in the central few parsecs of our galaxy, J. Korean Astron. Soc. 42, 17 (2009).

[45] R. Schdel, A. Feldmeier, D. Kunneriath, S. Stolovy, N. Neumayer, P. Amaro-Seoane, and S. Nishiyama, Surface brightness profile of the Milky Ways nuclear star cluster, Astron. Astrophys. 566, A47 (2014).

[46] S. Chatzopoulos, T. Fritz, O. Gerhard, S. Gillessen, C. Wegg, R. Genzel, and O. Pfuhl, The old nuclear star cluster in the milky way: Dynamics, mass, statistical parallax, and black hole mass, Mon. Not. R. Astron. Soc. 447, 948 (2015).

[47] T. Fritz, S. Chatzopoulos, O. Gerhard, S. Gillessen, R. Genzel, O. Pfuhl, S. Tacchella, F. Eisenhauer, and T. Ott, The nuclear cluster of the milky way: Total mass and luminosity, Astrophys. J. 821, 44 (2016).

[48] Y. Sofue, M. Honma, and T. Omodaka, Unified rotation curve of the galaxydecomposition into de vaucouleurs bulge, disk, dark halo, and the 9-kpc rotation dip, Publ. Astron. Soc. Jpn. 61, 227 (2009).

[49] Y. Sofue, A grand rotation curve and dark matter halo in the Milky Way Galaxy, Publ. Astron. Soc. Jpn. 64, 75 (2012).

[50] Y. Sofue, Rotation curve and mass distribution in the galactic center-From black hole to entire galaxy, Publ. Astron. Soc. Jpn. 65, 118 (2013).

[51] L. Chemin, F. Renaud, and C. Soubiran, The incorrect rotation curve of the milky way, Astron. Astrophys 578, A14 (2015).

[52] Y. Sofue, Dark halos of M-31 and the Milky Way, Publ. Astron. Soc. Jpn. 67, 75 (2015).

[53] M. Portail, O. Gerhard, C. Wegg, and M. Ness, Dynamical modelling of the galactic bulge and bar: The Milky Way's pattern speed, stellar and dark matter mass distribution, Mon. Not. R. Astron. Soc. 465, 1621 (2017).

[54] R. Launhardt, R. Zylka, and P. G. Mezger, The nuclear bulge of the galaxy. 3. Large scale physical characteristics of stars and interstellar matter, Astron. Astrophys. 384, 112 (2002).

[55] R. Schnrich, M. Aumer, and S. E. Sale, Kinematic detection of the galactic nuclear disk, Astrophys. J. Lett. 812, L21 (2015).

[56] C. Wegg, O. Gerhard, and M. Portail, The initial mass function of the inner galaxy measured from OGLE-III microlensing timescales, Astrophys. J. Lett. 843, L5 (2017).

[57] M. Persic, P. Salucci, and F. Stel, The Universal rotation curve of spiral galaxies: 1 . The dark matter connection, Mon. Not. R. Astron. Soc. 281, 27 (1996).

[58] L. M. Fernandez-Hernandez, M. A. Rodriguez-Meza, and T. Matos, Comparison between two scalar field models using rotation curves of spiral galaxies, J. Phys. Conf. Ser. 1010, 012005 (2018).

[59] H. Deng, M. P. Hertzberg, M. H. Namjoo, and A. Masoumi, Can light dark matter solve the core-cusp problem?, Phys. Rev. D 98, 023513 (2018).

[60] D. C. Rodrigues, A. del Popolo, V. Marra, and P. L. C. de Oliveira, Evidence against cuspy dark matter haloes in large galaxies, Mon. Not. R. Astron. Soc. 470, 2410 (2017).

[61] J. Veltmaat, J. C. Niemeyer, and B. Schwabe, Formation and structure of ultralight bosonic dark matter halos, Phys. Rev. D 98, 043509 (2018). 
[62] D. G. Levkov, A. G. Panin, and I. I. Tkachev, Bose Condensation by Gravitational Interactions, Phys. Rev. Lett. 121, 151301 (2018).

[63] J.-W. Lee, J. Lee, and H.-C. Kim, The M-sigma relation of super massive black holes from the scalar field dark matter, arXiv:1512.02351.

[64] J. Barranco, A. Bernal, J. C. Degollado, A. Diez-Tejedor, M. Megevand, M. Alcubierre, D. Nunez, and O. Sarbach, Are black holes a serious threat to scalar field dark matter models?, Phys. Rev. D 84, 083008 (2011).

[65] J. Barranco, A. Bernal, J. C. Degollado, A. Diez-Tejedor, M. Megevand, M. Alcubierre, D. Nunez, and O. Sarbach,
Schwarzschild Black Holes Can Wear Scalar Wigs, Phys. Rev. Lett. 109, 081102 (2012).

[66] J. Barranco, A. Bernal, J. C. Degollado, A. Diez-Tejedor, M. Megevand, D. Nunez, and O. Sarbach, Self-gravitating black hole scalar wigs, Phys. Rev. D 96, 024049 (2017).

[67] W. G. Unruh, Absorption cross-section of small black holes, Phys. Rev. D 14, 3251 (1976).

[68] R. Bender et al., Hst stis spectroscopy of the triple nucleus of m31: Two nested disks in keplerian rotation around a supermassive black hole, Astrophys. J. 631, 280 (2005). 